ARTICLE

\title{
The chromatin, topological and regulatory properties of pluripotency-associated poised enhancers are conserved in vivo
}

\author{
Giuliano Crispatzu (10) 1,2,3,8凶 , Rizwan Rehimi ${ }^{1,8}$, Tomas Pachano (i) 1, Tore Bleckwehl ${ }^{1}$, Sara Cruz-Molina (1) ${ }^{4}$, \\ Cally Xiao (1) 1,3,5,6, Esther Mahabir (10) 1, Hisham Bazzi (1) ${ }^{1,3,5}$ \& Alvaro Rada-Iglesias (1) 1,3,7凶
}

Poised enhancers (PEs) represent a genetically distinct set of distal regulatory elements that control the expression of major developmental genes. Before becoming activated in differentiating cells, PEs are already bookmarked in pluripotent cells with unique chromatin and topological features that could contribute to their privileged regulatory properties. However, since PEs were originally characterized in embryonic stem cells (ESC), it is currently unknown whether PEs are functionally conserved in vivo. Here, we show that the chromatin and 3D structural features of PEs are conserved among mouse pluripotent cells both in vitro and in vivo. We also uncovered that the interactions between PEs and their target genes are globally controlled by the combined action of Polycomb, Trithorax and architectural proteins. Moreover, distal regulatory sequences located close to developmental genes and displaying the typical genetic (i.e. CpG islands) and chromatin (i.e. high accessibility and H3K27me3 levels) features of PEs are commonly found across vertebrates. These putative PEs show high sequence conservation within specific vertebrate clades, with only a few being evolutionary conserved across all vertebrates. Lastly, by genetically disrupting PEs in mouse and chicken embryos, we demonstrate that these regulatory elements play essential roles during the induction of major developmental genes in vivo.

\footnotetext{
${ }^{1}$ Center for Molecular Medicine Cologne (CMMC), University of Cologne, Cologne, Germany. ${ }^{2}$ Department of Internal Medicine II, University Hospital Cologne, Cologne, Germany. ${ }^{3}$ Cluster of Excellence for Aging Research (CECAD), University of Cologne, Cologne, Germany. ${ }^{4}$ Max Planck Institute for Molecular Biomedicine, Muenster, Germany. ${ }^{5}$ Department of Dermatology and Venereology, University Hospital Cologne, Cologne, Germany. ${ }^{6}$ Laboratory of Neuro Imaging, USC Stevens Neuroimaging and Informatics Institute, Keck School of Medicine of University of Southern California, Los Angeles, CA, USA. ${ }^{7}$ Instituto de Biomedicina y Biotecnología de Cantabria (IBBTEC), CSIC-Universidad de Cantabria-SODERCAN, Santander, Spain. ${ }^{8}$ These authors contributed equally: Giuliano Crispatzu, Rizwan Rehimi.凶email: gcrispat@uni-koeln.de; alvaro.rada@unican.es
} 
P oised enhancers (PEs) were originally described in $\mathrm{ESC}^{1,2}$ as a rather limited set of distal regulatory elements that, before becoming activated upon cellular differentiation, are already bookmarked in pluripotent cells with unique chromatin and topological features. Briefly, in ESC, PEs are already bound by transcription factors and co-activators (e.g. p300), display high chromatin accessibility and are marked with H3K4me1, which are all typical features of active enhancers (Supplementary Fig. 1A). However, in contrast to active enhancers, PEs are not marked with H3K27ac, but are bound instead by Polycomb Group protein complexes $\left(\mathrm{PcG}^{3}\right)$ and their associated histone modifications (e.g. H3K27me3). Moreover, it was previously reported that PEs can physically interact with their target genes already in $\mathrm{ESC}^{4}$ in a PcG-dependent manner ${ }^{5}$. Most importantly, PEs were shown to be essential for the proper induction of their target genes upon differentiation of ESC into Anterior Neural Progenitor Cells $(A n t N P C)^{5}$. The previous epigenetic and topological features could explain, at least partly, why PEs are essential for the induction of major developmental genes. More recently, we also showed that the unique epigenetic, topological, and regulatory properties of PEs are genetically encoded and critically dependent on the presence of CpG islands (CGIs) that are not associated with annotated promoters and that are referred to as orphan CGI $(\mathrm{oCGI})^{6-9}$. Altogether, this led us to suggest that the genetic, epigenetic, and topological features of PEs could endow developmental loci with a permissive regulatory landscape that facilitates the precise and specific induction of PE-target genes upon pluripotent cell differentiation ${ }^{5,9}$. However, the previous characterization of PEs was based on the analyses of a few loci in which ESC were used as an in vitro differentiation model. Therefore, it is still unclear whether PEs exist and display essential regulatory functions in vivo.

By generating and mining various types of genomic data, here we show that PEs display their characteristic genetic, epigenetic, and topological features in both in vitro and in vivo pluripotent cells. Furthermore, we also show that PEs are pervasively found across vertebrates, although they tend to be preferentially conserved within specific vertebrate clades. Chiefly, by deleting conserved PEs in mouse and chicken embryos, we conclusively demonstrate that this type of regulatory elements is essential for the proper expression of major developmental genes during vertebrate embryogenesis.

\section{Results}

Poised enhancers (PEs) display their characteristic chromatin signature in the mouse post-implantation epiblast. Poised, active, and primed enhancers were previously identified in mESC grown under serum + LIF $(S+L)$ conditions ${ }^{5}$, which only recapitulates part of the pluripotency states that exist both in vitro and in vivo ${ }^{10,11}$. Therefore, to gather a more complete view of the full repertoire of pluripotency-associated PEs, we analyzed the necessary data (i.e. ATAC-seq, H3K27ac ChIP-seq, H3K27me3 ChIP-seq, H3K4mel ChIP-seq) to identify these regulatory elements in $2 \mathrm{i}$ mESC (naïve pluripotency) and EpiLC (epiblast-like cells; formative pluripotency) ("Methods"; Supplementary Data 1). Next, PEs identified in S + L mESC, 2i mESC, and EpiLC were combined, resulting in a total of 4191 unique mouse PEs (Fig. 1a; Supplementary Fig. 1A-C). A subset of these PEs, which we refer to as PoiAct enhancers $(n=354)$, gets activated in AntNPC as they overlap H3K27ac peaks identified in these cells ${ }^{5}$ (Fig. 1a). A similar strategy was used to identify active (high chromatin accessibility/p300 binding, H3K27ac+; $n=14803$ ) and primed enhancers (H3K4me1+, H3K27ac-, H3K27me3-; $n=55812)$ in the different mouse in vitro pluripotent cell types ("Methods"; Supplementary Fig. 1A-C). Once these combined enhancer sets were identified, we investigated their epigenetic profiles during early mouse development (i.e. fertilization (E0) to gastrulation (E6.5)). To this purpose, we mined publically available ATAC-seq and H3K27me3 ChIP-seq data sets ${ }^{12-14}$ and also generated relevant data in mouse E6.5 epiblast (i.e. ATAC-seq, $\mathrm{H} 3 \mathrm{~K} 27 \mathrm{ac}$ ChIP-seq). Regarding H3K27me3 levels at PEs, we found that this histone modification is especially high in sperm ${ }^{14,15}$, while it progressively accumulates during oocyte development (Supplementary Fig. 2A). Subsequently, H3K27me3 becomes erased upon fertilization and then it progressively increases until it reaches high levels in the post-implantation epiblast (E5.5-6.5) (Fig. 1b), thus resembling how this histone modification accumulates at bivalent promoters ${ }^{14}$. Similarly, chromatin accessibility (i.e. measured by ATAC-seq) progressively increases at PEs following fertilization, reaching its highest levels in the post-implantation epiblast (E6.5) (Fig. 1b). Therefore, the chromatin signature that PEs display in mESC (i.e. high chromatin accessibility and $\mathrm{H} 3 \mathrm{~K} 27 \mathrm{me} 3$ ) is also observed in vivo in pluripotent cells from the post-implantation epiblast. Importantly, the PEs remain inactive in the post-implantation epiblast, as they display low H3K27ac (Fig. 1c). In contrast, and in agreement with our previous observations, the PEs became active and, thus, gained $\mathrm{H} 3 \mathrm{~K} 27 \mathrm{ac}$ at later developmental stages (e.g. E12.5), particularly in the developing brain (Supplementary Fig. 2B). To further illustrate the existence of PEs in vivo, we used ATAC-seq and ChIP-seq data generated in the mouse E6.5 epiblast ("Methods") to directly call PEs in vivo (Fig. 1c). Out of the 3057 PEs identified in the E6.5 epiblast, 39.68\% (1213/3057) overlapped with the PEs identified in the in vitro pluripotent cells and $41.09 \%(1256 / 3057)$ became activated in the E10.5 brain (i.e. in vivo PoiAct enhancers). On the other hand, 30.06\% (1260/ 4191 ) of the in vitro PE were also called in vivo. Considering the current limitations to generate high-quality ChIP-seq data in the mouse epiblast, these results further support the presence of PEs in pluripotent cells both in vitro and in vivo.

Mouse PEs display high genetic and epigenetic conservation across mammals. Having confirmed that PEs display their characteristic chromatin features in vivo, we then assessed their evolutionary conservation among vertebrate genomes ("Methods"; Fig. 1d; Supplementary Fig. 2C, D), as this can provide preliminary insights into the functional relevance of non-coding sequences $^{16}$. In general, mouse PEs display high sequence conservation across mammals, which then decreases in nonmammalian amniotes (i.e. birds and reptiles) and is already quite moderate in anamniotes (i.e. amphibians and fish) (Fig. 1d; Supplementary Fig. 2C, D). Furthermore, PEs are considerably more conserved than active enhancers across all vertebrates ( $\mathrm{FC}=1.86, p=2.98 \mathrm{e}-06$; two-sided Wilcoxon test), probably reflecting the potential involvement of PEs in highly conserved developmental processes (e.g. organogenesis, patterning) $)^{1,5}$. Notably, despite their high evolutionary conservation, PEs display a limited overlap with "ultraconserved non-regulatory elements" (uCNEs) ${ }^{17}$ (Supplementary Fig. 2E), which, at least in some cases, act as enhancers of important developmental genes ${ }^{18,19}$. This suggests that, despite their overall high sequence conservation, PEs and uCNEs represent distinct classes of distal regulatory elements.

Having shown that mouse PEs display high genetic conservation, we then wanted to evaluate whether their unique chromatin signature (i.e. high chromatin accessibility/p300 binding, high $\mathrm{H} 3 \mathrm{~K} 27 \mathrm{me} / \mathrm{PcG}$ binding) was evolutionary conserved. To this end, we mined ATAC-seq and ChIP-seq data previously generated in ESC from different mammals (human ${ }^{1}$, chimp $^{20}$ ), as well as in early stages of zebrafish development ${ }^{21}$. In addition, 
a
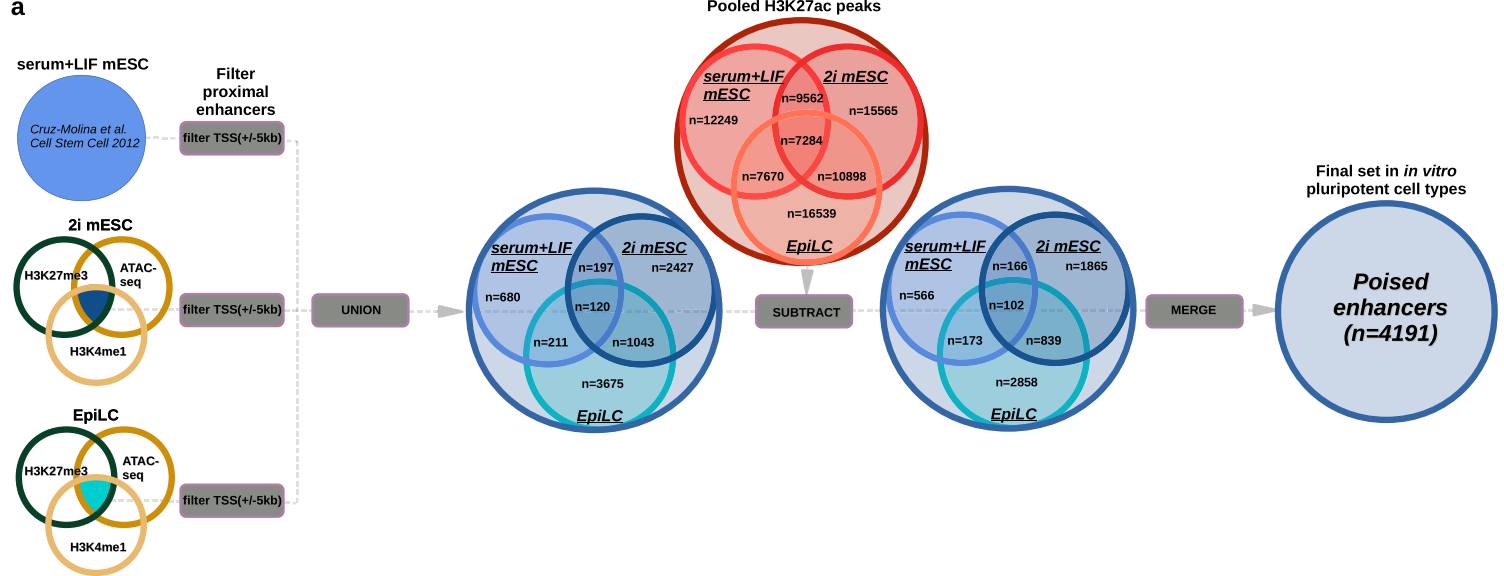

fitter TSS(+1-5kb)

b

Epigenomic signature of PEs identified in in vitro pluripotent cells during early mouse

C PEs in mouse E6.5 epiblast $(n=3057)$
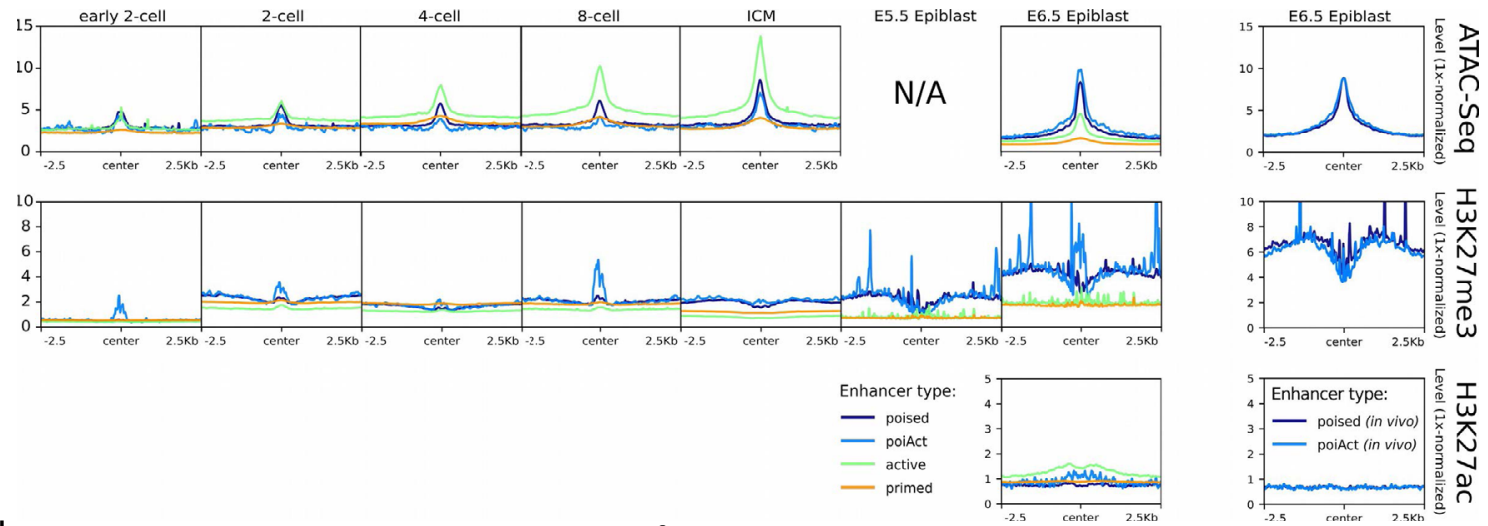

e

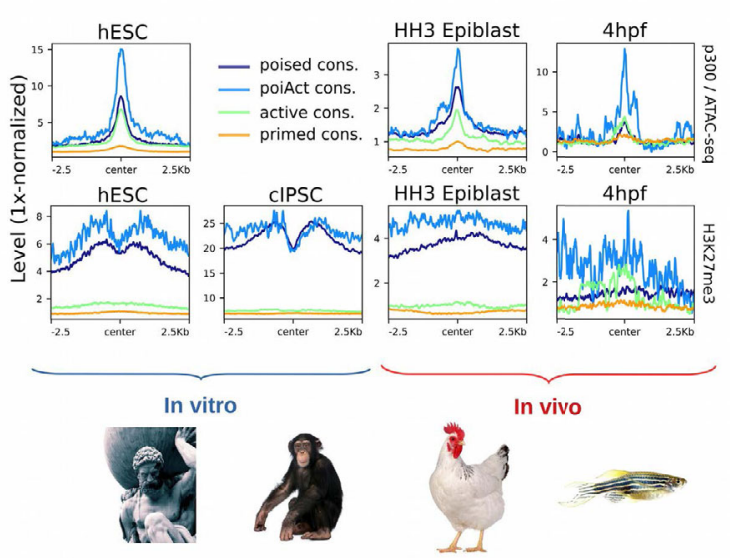

\section{,}

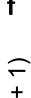

ร

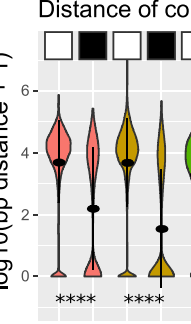

we also generated ATAC-seq and H3K27me3 ChIP-seq data for the chicken epiblast (HH3). Next, we analyzed the chromatin features of the mouse PEs that were conserved in each of the previous vertebrate species (Fig. 1e). In mammalian ESC and to a lesser extent in the chicken epiblast, PEs showed the expected chromatin signature (i.e. high H3K27me3 and ATAC-seq levels), while this was less obvious in zebrafish embryos, especially for H3K27me3. There are probably several reasons contributing to these differences: (i) the conserved PEs in non-mammalian vertebrates might include TF binding sites (indirectly detected by p300/ATAC peaks) but not nearby oCGI, which are responsible for PcG recruitment and H3K27me3 enrichment ${ }^{9}$ (see below); (ii) 
Fig. 1 Mouse poised enhancers (PEs) display their characteristic chromatin signature in vivo and are highly conserved in mammals. a ATAC-seq and ChIP-seq data for p300, H3K4me1, H3K27me3, and H3K27ac were used to call PEs in three distinct in vitro pluripotent states (i.e. 2i ESC, S + L ESC and EpiLC) ${ }^{5,94}$. PEs are characterized by high p300/ATAC signals, high H3K27me3, and H3K4me1 levels, as well as low H3K27ac levels and located at least 5 kb apart from a TSS ("Methods"). b ATAC-seq, H3K27ac, and H3K27me3 signals during early mouse embryogenesis ${ }^{12-14}$ (2-cell stage to E6.5 epiblast) are shown around poised, active, primed and PoiAct enhancers identified in in vitro pluripotent cells. c Public available H3K27me ${ }^{14}$, as well as generated ATAC-seq and H3K27ac signals during early mouse embryogenesis (E6.5 epiblast) are shown around poised and PoiAct enhancers identified in in vivo. $\mathbf{d}$ Sequence conservation across 68 vertebrate species (Supplementary Data 1) was measured for poised, active, primed, and PoiAct enhancers using a mappability threshold of 0.5 . PEs and PoiAct enhancers show significantly higher conservation than active $(\mathrm{FC}=1.86, p=2.98 \mathrm{e}-06 ; \mathrm{FC}=2.2, p=5.25 \mathrm{e}$ -08 respectively; two-sided Wilcoxon test) or primed enhancers ( $F C=1.72, p=4.44 \mathrm{e}-06$; $F C=2.03, p=9.61 \mathrm{e}-08$ respectively; two-sided Wilcoxon test). e ATAC-seq and H3K27me3 signals from human ESC (hESC), chimpanzee iPSC (cIPSC), epiblast from HH3 chicken embryos and 4 hpf zebrafish embryos are shown around poised, active, primed and PoiAct enhancers identified in mouse pluripotent cells and conserved in each of the corresponding vertebrate species. $\mathbf{f}$ Distance between active or poised mouse enhancers conserved in the indicated vertebrate species and CGIs identified in the same species using Bio-CAP6, 22. The asterisks indicate that PEs are significantly closer to CGls than active enhancers in all species except the frog (two-sided Wilcoxon test). The exact overlap of PEs (compared to active enhancers) with CGIs increases in all these species: in human from $9.85 \%$ to $43.14 \%$ $(p=1.18 \mathrm{e}-276)$, in mouse from $10.88 \%$ to $58.91 \%(p<2.2 \mathrm{e}-16)$, in platypus from $13.09 \%$ to $29.68 \%$ ( $p=2.51 \mathrm{e}-06$ ), in chicken from $9.93 \%$ to $25.95 \%$ $(p=1.37 \mathrm{e}-14)$, in lizard from $3.23 \%$ to $10.56 \%(p=0.017)$, in Xenopus tropicalis from $3.90 \%$ to $8.72 \%(p=0.94)$, and in zebrafish from $2.99 \%$ to $9.07 \%$ $(p=0.23) .{ }^{\star} p \leq 0.05,{ }^{\star \star} p \leq 0.01,{ }^{\star \star \star} p \leq 0.001,{ }^{\star \star \star \star} p \leq 0.0001$

the lower number of conserved PEs in non-mammalian vertebrates (chicken $n=767$, zebrafish $n=354$ ) can make the ATAC-seq and ChIP-seq profiles to look noisier; (iii) the data for non-mammalian vertebrates was generated in vivo, which due to lower cell numbers and higher cellular heterogeneity can also compromise the quality of the ATAC-seq and ChIP-seq profiles. Nevertheless, even in non-mammalian vertebrates (i.e. chicken and zebrafish), the conserved PEs display ATAC-seq and H3K27me3 signals clearly higher than those observed for other enhancer classes.

PEs are a prevalent feature of vertebrate genomes. We previously found that PEs have a unique and modular genetic composition $^{5,9}$, since compared to other enhancer classes, they are frequently located close to oCGI (i.e. $70-80 \%$ of PEs are located within $3 \mathrm{~kb}$ of computationally defined "weak" CGIs ${ }^{5}$ or biochemically defined CGIs", also known as Non-Methylated Islands $(\mathrm{NMI})^{22}$ ). Our previous work based on the in-depth analyses of a few selected PE loci suggests that the proximity to CGI confers PEs with unique epigenetic features, such as binding by PcG and DNA hypomethylation $5,9,23-25$. Congruently, analysis of whole-genome bisulfite sequencing data generated in mouse pluripotent cells ${ }^{26,27}$ revealed that PEs are globally hypomethylated both in vitro and in vivo (Supplementary Fig. 3A). Moreover, PEs are bound in mESC by KDM2B (Supplementary Fig. 3B), a protein containing CXXC domains that specifically recognize CGI and that might be responsible, at least partly, for the unique epigenetic properties of the PEs ${ }^{24,28,29}$ (Supplementary Fig. 3C). Given the importance that CGI might have in conferring PEs with their unique chromatin and regulatory features, we then wanted to evaluate whether the proximity between PEs and CGI was evolutionary conserved. However, CGIs have been traditionally identified using algorithms originally implemented in mammalian genomes, but that, due to the variability in overall GC and CpG contents, do not perform well when applied to cold-blooded vertebrate genomes. To overcome these limitations, we used data previously generated in seven different vertebrates by Bio-CAP (biotinylated CxxC-affinity purification ${ }^{6,22}$ ), an assay that enables the unbiased identification of CGI. The CGIs identified through Bio-CAP are typically referred to as $\mathrm{NMI}^{22}$. However, to avoid possible confusions, from now on we will simply use the term CGI regardless of whether these genetic features were identified based on their genetic composition or by Bio-CAP. Next, we measured the distance between those mouse PEs conserved in each vertebrate species and the nearest CGI (Fig. 1f; Supplementary Fig. 3D). Among the seven vertebrate species, the proximity of PEs to CGIs was particularly obvious in mammals (mouse, human, platypus) and, to a lesser extent, in chicken. Similarly, we observed that conserved PEs tended to be considerably closer to CGIs than their active counterparts in both mammals and chicken, but not in anamniotes (i.e. frog and zebrafish) (Fig. 1f). Therefore, considering the important role of oCGI in mediating the recruitment of PcG to PEs in $\mathrm{mESC}^{9}$, the weaker $\mathrm{H} 3 \mathrm{~K} 27 \mathrm{me} 3$ enrichment observed at conserved PEs in zebrafish embryos (Fig. 1e) might be explained, at least partly, by the frequent absence of nearby oCGI.

Together with our sequence conservation analyses, the previous results suggest that PEs are prevalent in mammals but rather scarce in other vertebrates, especially in anamniotes. Alternatively, PEs might be abundant in all vertebrates, but sequence conservation, including the proximity to oCGI, might preferentially occur within individual vertebrate clades. To distinguish between these two possibilities, we called PEs de novo in human ESC, chicken epiblast and zebrafish embryos using available epigenomic data sets $1,21,30$ (Fig. 2a) and similar criteria to the ones used to identify PEs in mouse cells (Fig. 1a). The term de novo is used to define PEs that are directly identified using epigenomic data generated in each of the investigated vertebrate species in contrast to those solely defined by sequence conservation (Fig. 1d-f). Notably, these de novo PEs were abundant in both mammals (human $n=4009$ ) and nonmammals (chicken $n=7306$, zebrafish $n=2534$ ) and, especially in zebrafish, they displayed stronger ATAC-seq and H3K27me3 signals than PEs identified through conservation analyses (Fig. 2a; Fig. 1e). Accordingly, while in human ESC and chicken epiblast both de novo and conserved PEs were similarly close to CGIs, in the zebrafish embryos the proximity to CGIs was dramatically increased for the de novo PEs (Fig. 2b). Furthermore, in all the investigated species, the de novo PE was strongly associated with genes involved in developmental processes, such as patterning and organogenesis (Fig. 2c; Supplementary Fig. 3E, F). Overall, these results indicate that PEs showing similar genetic (i.e. proximity to oCGI) and epigenetic (i.e. high ATAC/p300 and H3K27me3 levels) features are prevalent in all vertebrates, but that a large fraction of them might be specific to each vertebrate class/group. In agreement with this possibility, chicken and zebrafish de novo PEs were highly conserved among birds/reptiles and fish, respectively, but not in other vertebrates (Fig. 2d), thus resembling the preferential conservation of mouse PE within mammals (Fig. 1d). Nevertheless, we also noticed that those de novo PE showing high sequence conservation tended to be even closer to CGIs (Supplementary Fig. 3G) and, displayed higher 
a
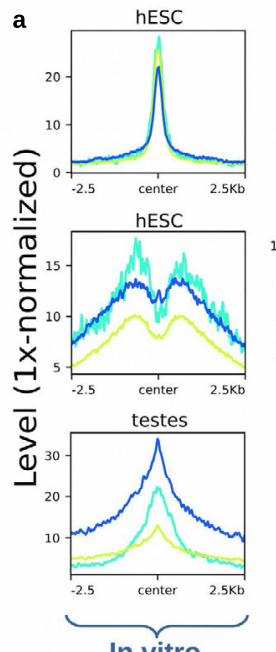

In vitro
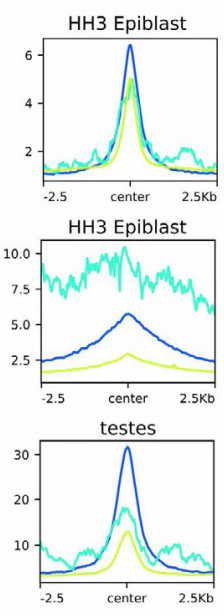
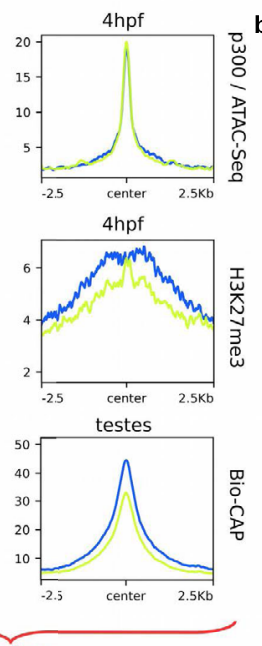

In vivo

$=\quad$ Distance to de novo poised enhancers

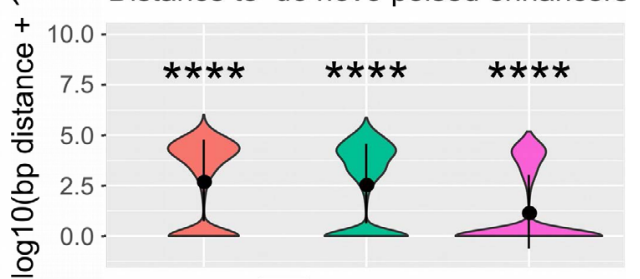

Human (hg19)

Chicken (galGal6)

Danio rerio (danRer10)

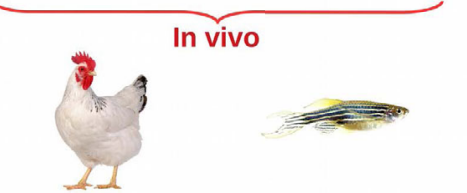

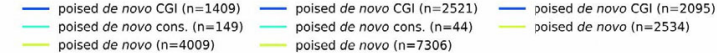

c
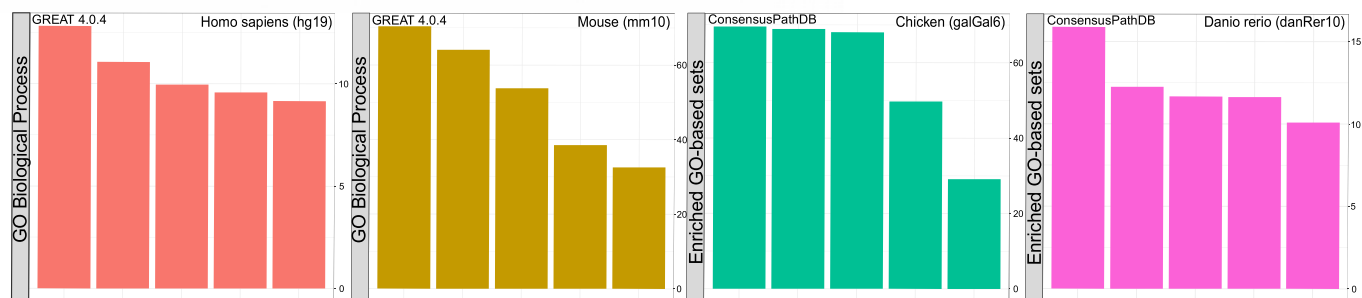

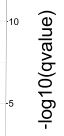
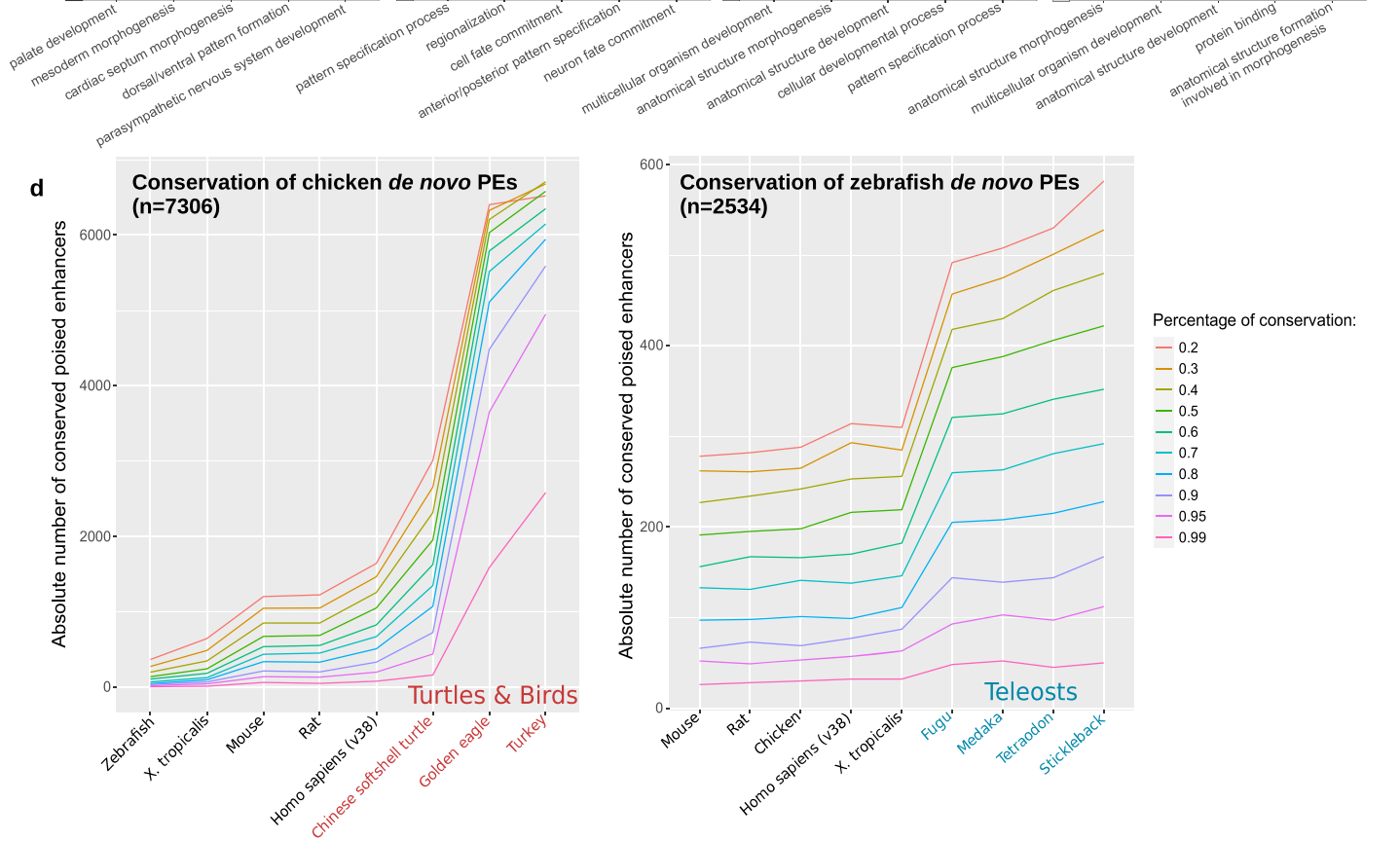

$-0.2$

$-0.3$

$-0.4$

$-0.6$

-0.7
-0.8

$-0.9$

-0.95
-0.99

H3K27me3 levels (Fig. 2a), which according to our recent work ${ }^{9}$, might endow PEs with particularly privileged regulatory properties.

PEs globally interact with bivalent gene promoters in pluripotent cells both in vitro and in vivo. The presence of CGI in the vicinity of PEs provides them with unique topological properties. Namely, PEs can already physically interact with their target genes in ESC, thus preceding their activation in neural progenitors $^{5,9}$. This might confer anterior neural loci with a permissive regulatory topology that facilitates the precise and robust induction of PE-target genes. However, this model is based on the detailed analysis of a few endogenous (i.e. Sox1, Six3, $L m \times 1 b, L h \times 5)$ or ectopic (i.e. Gata6) PE loci using 4C-seq 
Fig. 2 PEs are a widespread feature across vertebrates. a PEs were called de novo using available ATAC-Seq, p300 ChIP-seq and H3K27me3 ChIP-Seq data generated in human embryonic stem cells (hESC), HH3 chicken epiblast and zebrafish 4hpf zebrafish embryos (sphere stage; blastulation). ATACseq, ChIP-seq, and Bio-CAP6,22 signals are shown for each species around all de novo PEs as well as those overlapping CGIs or previously called conserved mouse PEs (except for zebrafish due to low numbers; $n=2$ ). b Distance between de novo PEs and CGIs for each of the investigated vertebrate species. The asterisks indicate that de novo PEs are significantly closer to CGIs than active mouse enhancers conserved in each species. The exact overlap of de novo PEs (compared to active enhancers) with CGIs increases in all species: in human from $9.85 \%$ to $33.18 \%$ ( $p=6.64 \mathrm{e}-69$; two-sided Wilcoxon test); in chicken from $9.93 \%$ to $34.15 \%$ ( $p=9.88$ e-27; two-sided Wilcoxon test) and in zebrafish from $2.99 \%$ to $68.77 \%$ ( $p=5.57$ e-61; two-sided Wilcoxon test). c De novo PE sets were annotated using GREAT 4.0.4 ${ }^{97}$ for human and mouse. As this software is not available for chicken and recent zebrafish versions, these de novo PEs were linked to the nearest gene and the resulting gene set was analyzed using ConsensusPathDB ${ }^{98}$. $\mathbf{d}$ The sequence conservation of de novo PEs identified in chicken (left panel) and zebrafish (right panel) was measured across representative species of the main vertebrate clades using different mappability thresholds (mapping ratios $0.2-0.99$; color legend). ${ }^{\star} p \leq 0.05,{ }^{\star \star} p \leq 0.01,{ }^{\star \star \star} p \leq 0.001,{ }^{\star \star \star \star} p \leq 0.0001$.

technology 5,9 . In principle, the generality of our previous observations could be addressed using $\mathrm{Hi}-\mathrm{C}$ technology. However, to detect enhancer-promoter contacts at high resolution, $\mathrm{Hi}-\mathrm{C}$ typically requires large and cost-prohibitive sequencing depths ${ }^{31,32}$. Therefore, we decided to use a more targeted approach, called HiChIP ${ }^{33}$, that combines $\mathrm{Hi}-\mathrm{C}$ and $\mathrm{ChIP}$, thus allowing interrogation of DNA loops associated with proteins/ histone marks of interest. As both PEs and their target gene promoters are typically enriched in $\mathrm{H} 3 \mathrm{~K} 27 \mathrm{me} 3^{1,5}$, we first generated HiChIPs for $\mathrm{H} 3 \mathrm{~K} 27 \mathrm{me} 3$ in mESC grown under serum + LIF conditions as biological duplicates. Both replicates were pooled to call loops $(p<0.01 ; n=72265$; ranging from 25 $\mathrm{kb}-1.81 \mathrm{Mb}$ in loop size; "Methods"). In order to validate the quality of the previous HiChIP data and its usefulness to identify $\mathrm{PE}$-target gene interactions, we first evaluated the four PE loci previously analyzed by $4 \mathrm{C}$-seq and confirmed the reported contacts (Fig. 3a; Supplementary Fig. 4A). Moreover, when considering all the detected HiChIP loops, we found that a large fraction of distal PEs $(n=3239$; $>10 \mathrm{~kb}$ from a TSS) interacts with at least one locus $(55.7 \%)$. A large fraction of these interactions (39.8\%) were with gene promoters (Fig. 3 b), which, importantly, often display a bivalent state in mESC (Fig. 3c). More specifically, the chromatin state of 1083 out of the 1585 TSSs interacting with distal PEs in mESC (Fig. 3c) has been previously described ${ }^{34}$ and, among them, 424 are bivalent $(n=424 / 2794 ; p<2.2 \mathrm{e}-16$; OR $=$ 2.63; Fisher test), 40 are only marked with H3K27me3 $(n=40 /$ $160 ; p=8.88 \mathrm{e}-14 ; \mathrm{OR}=4.90$; Fisher test), 480 are only marked with H3K4me3 ( $n=480 / 9663 ; p=2.64 \mathrm{e}-06$; OR $=0.77$; Fisher test) and 155 are unmarked $(n=155 / 4758 ; p<2.2 \mathrm{e}-16$; OR $=$ 0.495 ; Fisher test). In agreement with this significant overrepresentation of bivalent and $\mathrm{H} 3 \mathrm{~K} 27$ me3-only genes, the genes interacting with PEs tend to be preferentially involved in developmental processes (e.g. patterning, morphogenesis) (Fig. 3d). Interestingly, we noticed that in contrast to the frequent longrange/inter-TAD interactions established between PcG-bound genes/domains $3,4,35, \mathrm{PE}$-gene contacts preferentially occur within the same TAD (i.e. intra-TAD) (Fig. 3e). Moreover, the interactions between PEs and bivalent genes detected by HiChIP $(n=$ $526)$ were also readily observed in $\mathrm{Hi}-\mathrm{C}$ data generated in $\mathrm{mESC}$ grown under both serum + LIF and $2 \mathrm{i}$ conditions ${ }^{36}$ (Fig. $3 f$ ). This further validates the quality of our HiChIP data and supports that $\mathrm{PE}$-gene contacts are present across different in vitro pluripotent states (Supplementary Fig. 1A). Once these pre-formed contacts between PEs and their target genes in mESC were globally confirmed, we also investigated whether this topological feature could be also observed in vivo using $\mathrm{Hi}-\mathrm{C}$ data recently generated in peri-implantation mouse embryos (E3.5-E7.5) ${ }^{37}$. Notably, we observed clear contacts between PEs and bivalent promoters in the mouse ICM as well as in the post-implantation epiblast (Fig. 3g). This is in agreement with the overall conservation of the PEs chromatin features in vivo (Fig. 1b, c), since such features, namely $\mathrm{H} 3 \mathrm{~K} 27 \mathrm{me} 3 / \mathrm{PcG}$, are considered as important mediators of $\mathrm{PE}-$ gene interactions ${ }^{5}$.

The physical communication between PEs and their target genes depends on the combined action of Polycomb, Trithorax, and architectural proteins. There is some discrepancy regarding which PcG complexes, PRC1 or PRC2, contribute to the physical communication between PcG-bound loci, including PE-target gene interactions $4,5,38-40$. To investigate whether PRC1 and/or PRC2 are globally involved in the establishment of PE-target gene interactions in mESC we used PRC2 $\left(\mathrm{EED}^{-1-} \mathrm{mESC}^{41}\right)$ and PRC1 (RING1a ${ }^{-/-}$RING1b $^{\mathrm{fl} / \mathrm{fl}} \mathrm{mESC}^{42}$ treated with Tamoxifen for $72 \mathrm{~h}$ ) null mESC lines (Supplementary Fig. 4B). In contrast to H3K27me3, which is globally lost in PRC1 and PRC2 null $\mathrm{mESC}^{5,43}$, H3K4me3 levels are maintained or even increased at gene promoters in general and bivalent ones in particular ${ }^{44}$ (Supplementary Fig. 4C), thus enabling the use of this histone mark to study PE-gene interactions in PcG-null ESC. Therefore, we generated $\mathrm{H} 3 \mathrm{~K} 4 \mathrm{me} 3 \mathrm{HiChIP}$ data as biological duplicates in each PcG-null mESC line. Overall, we observed that PRC1 null ESC showed a reduction in mid-range interactions and in the overall number of loops, probably reflecting the involvement of PRC1 in active enhancer-gene contacts ${ }^{40}$ (Fig. 4a). Most importantly, the contacts between PEs and bivalent promoters were globally reduced in PRC1 null cells (Fig. 4b). In contrast, in PRC2 null cells we observed strongly reduced $\mathrm{PE}$-gene interactions within previously investigated loci $^{5}$ (Fig. 4c; Supplementary Fig. 4D), but only mild effects at a global level (Fig. 4b). The importance of PRC1 for PE-gene interactions was also confirmed using $\mathrm{Hi}-\mathrm{C}$ data generated in different RING1A/B-depleted mESC lines (RING1a ${ }^{-/-}$RING1b ${ }^{\text {DEG }}$ mESC) (Fig. 4d). Therefore, our global analyses indicate that $\mathrm{PRC1}$ is required for proper PE-target gene communication in ESC. This is likely to be mediated by canonical PRC1 (cPRC1) through the polymerization and/or phase-separation capacity of its PHC and CBX subunits, respectively $34,45-49$.

It has been recently shown that the long-range interactions between PcG-bound genes/domains in mouse pluripotent cells are controlled not only by PRC1 but also by other protein complexes (i.e. Trithorax/MLL2, Cohesin $)^{50}$. Using Hi-C data generated in E6.5 epiblasts from $K m t 2 b^{k o}\left(M l l 2^{k o}\right)$ mice $^{51}$, we observed that the contacts between PEs and bivalent promoters were also reduced in the Kmt $2 b$ mutant epiblasts (Fig. $4 \mathrm{e}$ ). We then analyzed Hi-C data generated in a Scc1/Rad21-Degron ESC line ${ }^{52}$ and found that, while Cohesin depletion led, as previously reported ${ }^{52}$, to increased long-range interactions between PcG domains, it actually reduced intra-TAD contacts between PE and their bivalent target genes (Fig. 4d). Similarly, CTCF depletion ${ }^{53}$ also diminished the interactions between PE and bivalent genes (Fig. 4f). Therefore, PE-bivalent gene contacts might be the result of the combined action of two major mechanisms; (i) homotypic chromatin 
a
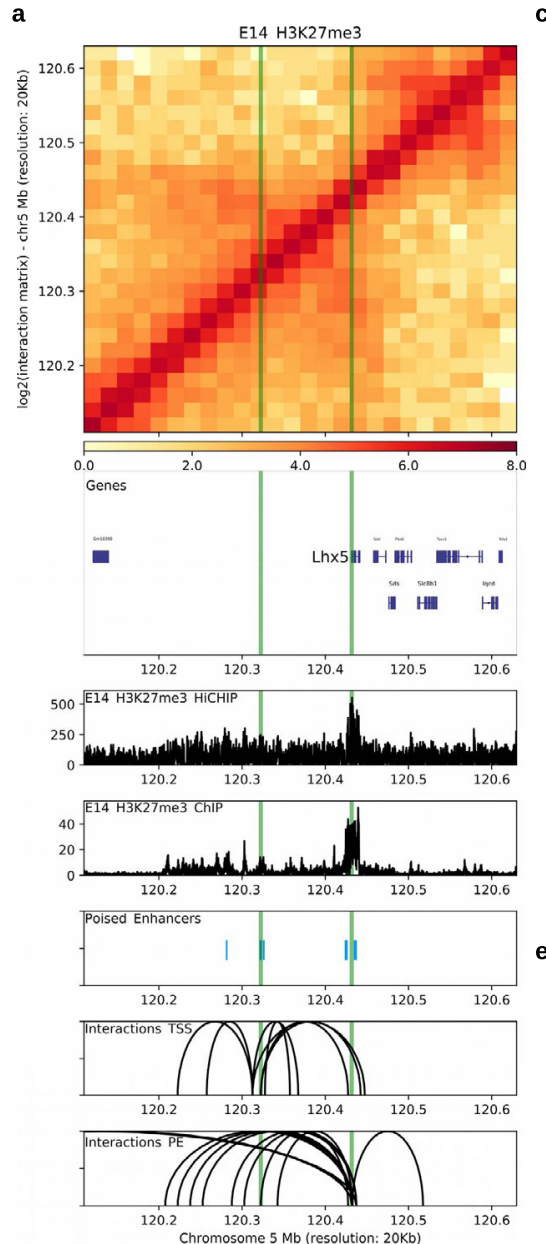

b

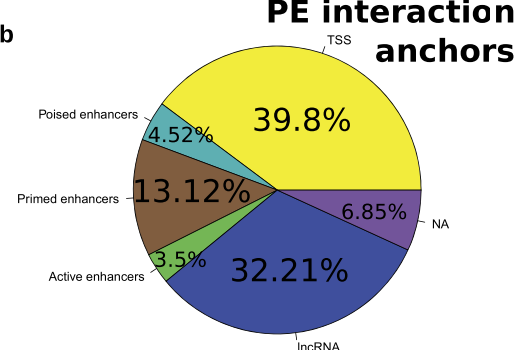

f

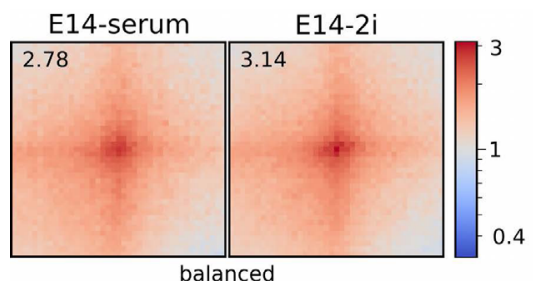

C $\mathrm{H} 3 \mathrm{~K} 27 \mathrm{me} 3 \mathrm{H} 3 \mathrm{~K} 37 \mathrm{ac}$

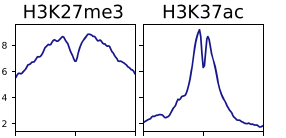

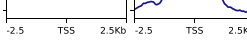
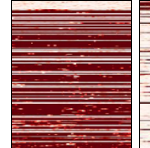

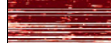

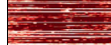

$\operatorname{lin}^{2}$
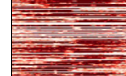

is

तin

ine

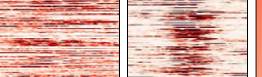

sin in

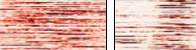

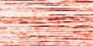

$-\quad-1$
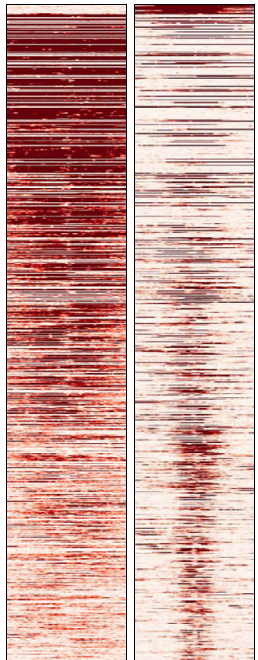

(1)
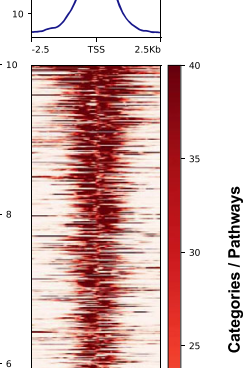

PcG-bound domains to any

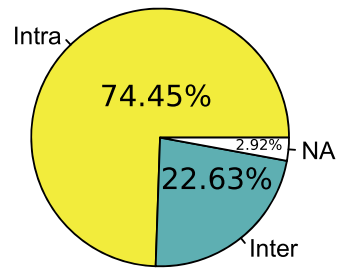

PE to general promoters

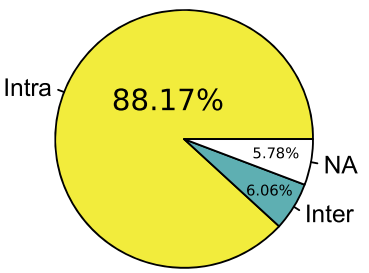

in cancer - Homo sapien

Neural Crest Differentiation

Endoderm Differentiatio

Activation of anterior HoX genes
in hindbrain develoment
during early embryogenes

尊

Dopaminergic Neurogeness

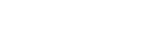

pattern specification process

multicellular organism developm

and

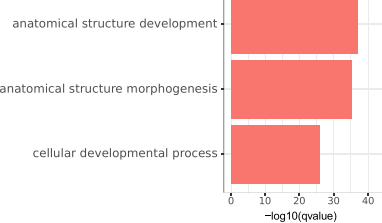

Enriched pathway-based sets
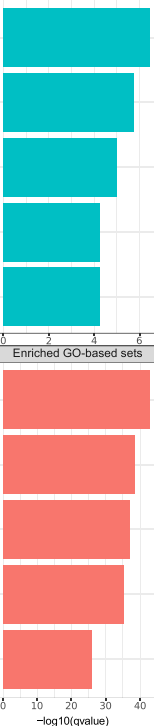

PcG-bound promoters to any
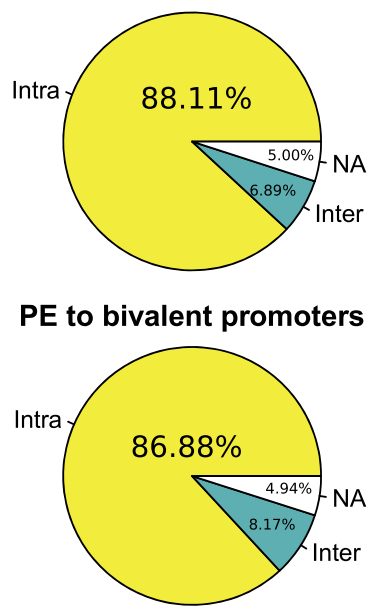

PE to bivalent promoters

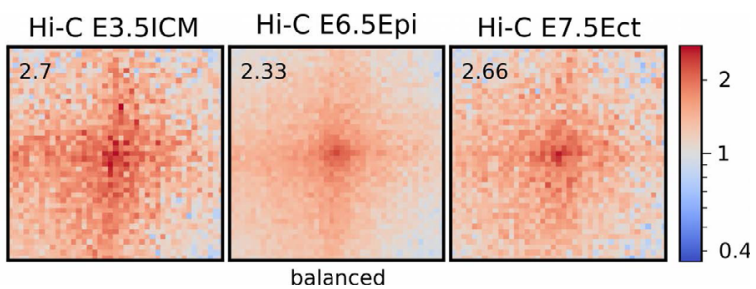

interactions mediated by PcG and Trithorax complexes and (ii) loop extrusion mechanisms dependent on Cohesin and CTCF that favor PE-gene communication within TADs (Fig. 3e).

The physical interactions between PEs and their target genes are maintained once PE get activated. Our previous analyses based on $4 \mathrm{C}$-seq experiments indicated that PE-gene contacts already present in ESC are maintained in AntNPC once the PE and their target genes become active. However, due to the cellular heterogeneity present within AntNPC, PEs and their target genes get activated only in a fraction of cells that cannot be specifically interrogated by $4 \mathrm{C}$-seq/Hi-C experiments. Therefore, we conducted H3K27ac HiChIP experiments in E14 AntNPC 54 and E10.5 mouse brains to specifically and globally evaluate 
Fig. 3 Mouse PEs globally interact with bivalent promoters in pluripotent cells both in vitro and in vivo. A H3K27me 3 HiChIP and ChIP-seq profiles generated in mESC are shown around the Lhx5 locus. Both the Lhx5 TSS and a PE previously shown to control this gene in AntNPC (PE Lhx5 ( $-109 \mathrm{~kb})$ ) are marked with green vertical lines. Upper panel: heatmap of log2 interaction intensities based on H3K27me3 HiChIP data generated in mESC. Two medium panels: ChIP-seq and 1D HiChIP signals for H3K27me3 in mESC. Lower two panels: significant $(p<0.01$; FitHiChIP102) interactions were called using the H3K27me3 HiChIP PETs (paired-end tags) and loops in which one of the anchors overlaps ( $\pm 10 \mathrm{~kb}$ ) either the PE Lhx5 ( $-109 \mathrm{~kb})$ or the Lhx5 TSS are shown. b Significant ( $p<0.01$; FitHiChIP) interactions were called using the mESC H3K27me3 HiChIP PETs (paired-end tags). Interaction anchors were overlapped with PEs and their interaction partners were hierarchically annotated with the categories shown in the pie chart ("Methods"). c mESC ChIP-seq profiles for H3K27me3, H3K27ac, and H3K4me3 are shown around the TSS $(n=1083)$ interacting with PEs according to the H3K27me3 HiChIP loops identified in mESC ( $p<0.01$; FitHiChIP). d Pathway (green) and Gene Ontology (red) analysis for the TSS/genes interacting with PEs according to the H3K27me3 HiChIP loops identified in mESC ( $p<0.01$; FitHiChIP) was performed with ConsensusPathDB. e The interactions (called using mESC H3K27me3 HiChIP data; $p<0.01$; FitHiChIP) established by PcG domains ("Methods") and PcG-bound promoters as well as those involving PE-general promoter or PEbivalent promoter pairs were classified as either intra-TAD (yellow) or inter-TAD (green) depending on whether the two anchors of each loop occur within the same or different TADs, respectively. $\mathbf{f}, \mathbf{g}$ ) Significant (mESC H3K27me3 HiChIP data; $p<0.01$; FitHiChIP) interactions between distal PEs $(>10 \mathrm{~kb}$ from TSS) and bivalent promoters $(n=526)$ were visualized as pileup plots using Hi-C data generated in vitro ${ }^{36}$ (2i ESC, serum+LIF ESC; $\mathbf{f}$ ) and in vivo ${ }^{37}$ (E3.5 Inner Cell Mass (ICM), E6.5 epiblast, E7.5 ectoderm; $\mathbf{g}$ ). Hi-C pairwise interactions are shown $250 \mathrm{~kb}$ up- and downstream of each PE and bivalent promoter pair. Hi-C interaction matrices were KR-balanced (balanced). The numbers in the upper left corners correspond to "loopiness" values (center intensity normalized to the intensity in the corners).

interactions established by $\mathrm{PE}$ that became active (i.e. PoiAct enhancers) in these cells. First, we evaluated the PE loci previously analyzed by $4 \mathrm{C}$-seq and confirmed that PE-target gene contacts are maintained once PE becomes active in both AntNPC and E10.5 brain cells (Fig. 5a, b; Supplementary Fig. 5A). More importantly, we found that distal PE-bivalent gene contacts detected in mESC (Fig. 3b) were also frequently observed once PEs became active in the developing mouse brain (50.76-54.56\% overlap; $n=67,748-94,731$ loops; $p<0.05 ; \pm 10 \mathrm{~kb}$ anchor extension) (Fig. 5 c) or in AntNPC (36.69\% overlap; $n=49,552$ loops; $p<0.05 ; \pm 10 \mathrm{~kb}$ anchor extension) (Fig. 5d). Moreover, distal PoiAct enhancer contacts were also found in AntNPC (55.90\% interacting with at least one locus) and the E10.5 brain (59.07-65.95\% interacting with at least one locus), while their target genes were induced in their respective differentiating and developmental stages (Supplementary Fig. 5B-D; Supplementary Fig. 6A, B).

Highly conserved PEs are necessary for the induction of major brain developmental genes in vertebrate embryos. The previous analyses show that the main genetic (e.g. proximity to CGI), chromatin (e.g. high $\mathrm{H} 3 \mathrm{~K} 27 \mathrm{me} 3 / \mathrm{PcG}$ levels), and topological (e.g. pre-formed contacts with target genes) features of PEs are evolutionary conserved and detectable in vivo. These observations support, albeit in a correlative manner, the functional relevance of PEs during early vertebrate embryogenesis, particularly during brain development. The functional relevance of PEs was previously demonstrated by deleting candidate PEs in mESC (i.e. $P E$ Lhx5(-109kb), PE Six3(-133kb), PE Sox1(+35kb), PE Wnt8b $(+21 \mathrm{~kb})$ ), which severely compromised the induction of major brain developmental genes (i.e. $L h x 5$, Six3, Sox1, Wnt8b) upon differentiation of ESC into AntNPCs ${ }^{5}$. However, it is currently unknown whether PEs also have essential and non-redundant regulatory functions in vivo or whether, alternatively, these privileged regulatory properties might represent an in vitro "artifact" due to the reduced robustness of in vitro differentiation systems. To start addressing this important question, we first used CRISPR/Cas9 technology to generate mouse embryos in which we deleted the PE Lhx5 $(-109 \mathrm{~kb})$, one of the PEs that we previously characterized in vitro (Fig. 6a-c; Supplementary Fig. 7A, B). Remarkably, the expression of $L h x 5$ was strongly reduced in the forebrain of E8.5 and E9.5 PE $\operatorname{Lh} \times 5(-109 \mathrm{~kb})^{-1-}$ mouse embryos in comparison to their WT isogenic controls (Fig. 6c). Next, since the mouse PE $L h \times 5(-109 k b)^{-/-}$has a high genetic and epigenetic conservation across vertebrates, we decided to generate targeted deletions of its homologous sequence in the developing brain of chicken embryos using CRISPR/Cas9 ${ }^{55}$ (Fig. 6c-e). Briefly, the forebrain of HH9 chicken embryos was unilaterally electroporated with vectors expressing Cas9 and gRNAs flanking the PE Lhx5 conserved sequence (Fig. 6d; Supplementary Fig. 7A, C). Subsequently, the expression of $L h x 5$ was evaluated by in situ hybridization (ISH) in HH14 chicken embryos ( to E11.5 in mice). Notably, the forebrain expression of Lhx 5 was strongly and specifically reduced in the electroporated side (Fig. 6f). Furthermore, the specificity of these results was further supported by experiments in which the electroporation of chicken embryos with Cas9 and scrambled gRNAs did not affect Lhx5 expression (Fig. 6f). To further evaluate the in vivo functional relevance of evolutionary conserved PEs, we similarly disrupted another two PEs (PE Six3(-133kb), PE Sox1 $(+35 k b))$ that were previously characterized in $\mathrm{mESC}^{5}$ and that were also genetically and epigenetically conserved in the chicken genome (Supplementary Fig. 7C-E). We again observed a strong reduction in the expression of Six 3 and Sox 1 in the electroporated side of embryos targeted with both Cas9 and gRNAs flanking the PEs, but not when Cas9 was electroporated with scrambled gRNAs (Fig. 6f). Furthermore, in the case of the PE Six3(-133kb) chicken homolog, its disruption resulted in a smaller and malformed eye, in agreement with the strong expression and conserved function of Six3 during eye development ${ }^{56}$ (Fig. 6f). Overall, these results demonstrate that the regulatory function of PEs is essential and conserved in vivo. However, whether the essential regulatory properties of these enhancers require a "poised" state previous to their full activation remains to be demonstrated.

\section{Discussion}

PEs were originally identified, mechanistically dissected, and functionally characterized in $\mathrm{ESC}^{1,5}$. Our previous work suggested that PEs could play essential roles during the induction of major developmental genes once pluripotent cells start differentiating ${ }^{5}$. However, it was still unclear whether PEs actually existed and were functionally relevant in vivo. Here we addressed this important question by mining various genomic and epigenomic datasets, which enabled us to conclusively show that PEs not only display their characteristic chromatin signature in vivo, but also that they are a prevalent feature of vertebrate genomes. Interestingly, we found that PEs tend to be highly conserved within specific vertebrate groups (e.g. mouse PEs are highly conserved across mammals; chicken PEs are highly conserved in birds and reptiles), while only a relatively small subset of PEs is conserved across all vertebrates. Nevertheless, in all the 


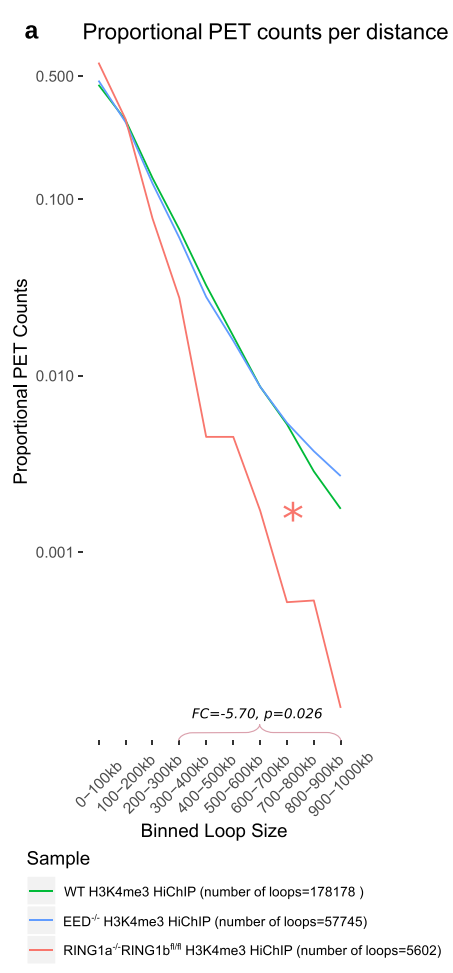

b

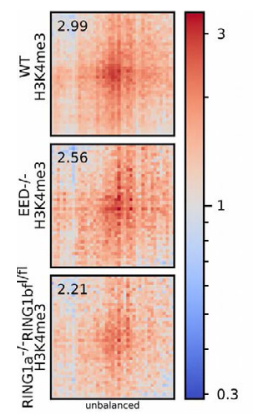

PE-gene contacts d

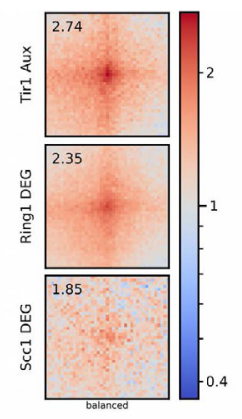

PE-gene contacts c

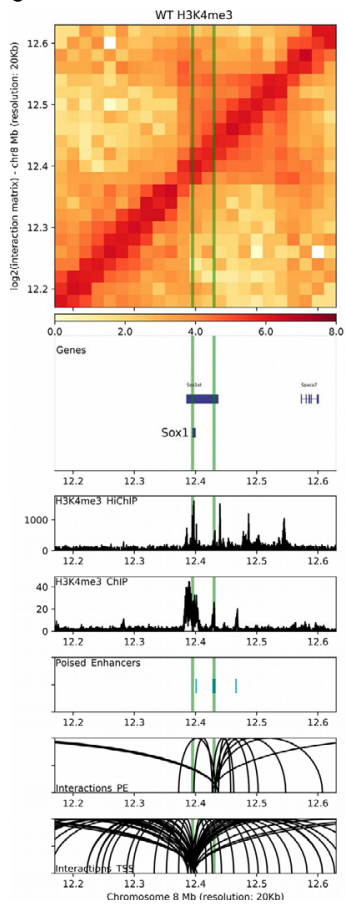

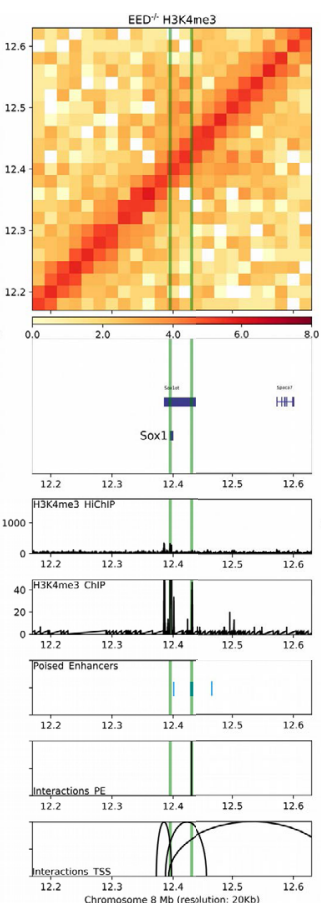

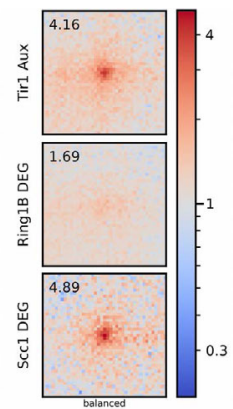

PcG-PcG contacts

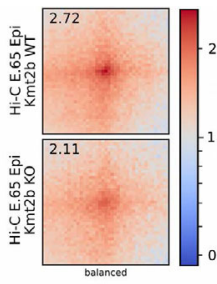

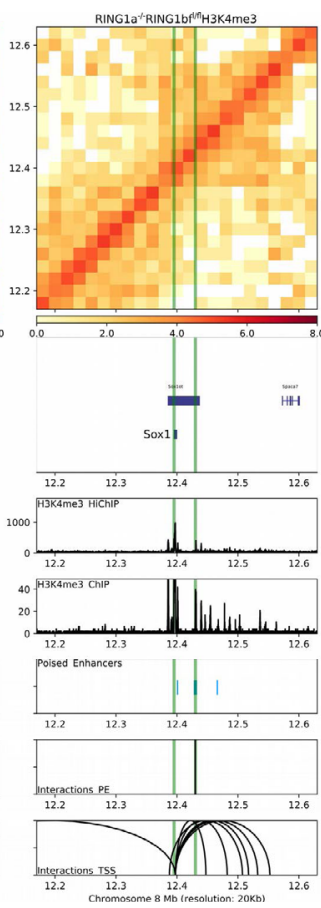

Fig. 4 Protein complexes controlling the interactions between PEs and bivalent promoters in mESC. a Significant ( $q<0.1 ;$ FitHiChIP) $\mathrm{H} 3 \mathrm{~K} 4 \mathrm{me} 3 \mathrm{HiChIP}$ interactions identified in WT mESC, Eed ${ }^{-/-}$mESC and Tamoxifen-treated Ring $1 a^{-/-R i n g} b^{f l / f l}$ mESC were plotted according to their loop size. Mid-range (300kb-1mb) loops are significantly reduced ( $F C=-5.70, p=0.026$; two-sided Wilcoxon test) in Tamoxifen-treated Ring $1 a^{-} /-$Ring 16 fl/fl $\mathrm{mESC}$ compared to WT. PET = paired-end tags. b Significant (mESC H3K27me3 HiChIP data; $p<0.01$; FitHiChIP) interactions between distal PEs ( $>10$ kb from TSS) and bivalent promoters $(n=526)$ were visualized as pileup plots using H3K4me3 HiChIP data generated in WT, Eed ${ }^{-/-}$and Tamoxifen-treated Ringla/-Ring $1 b^{f l / f l}$ mESC. HiChIP interaction matrices were coverage-normalized (unbalanced). c H3K4me3 HiChIP in WT, Eed-/- mESC, and Tamoxifen-treated

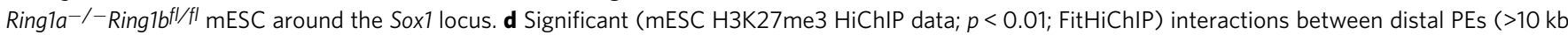
from TSS) and bivalent promoters (left panel; $n=526$ ) or between PcG domains (right panel; $n=411$ ) were visualized as pileup plots using Hi-C data generated in the following mESC lines expressing Tir1 and treated with Auxin 52 : WT mESC (Tir1 Aux), Ring1a-/-Ring1bDEG/DEG mESC (i.e. RING1A/Bdepleted cells; Ring1b Aux) and Rad21/Scc1DEG/DEG mESC (i.e. Cohesin depleted cells; Scc1 Aux). Hi-C interaction matrices were KR-balanced (balanced). e Significant (mESC H3K27me3 HiChIP data; $p<0.01$; FitHiChIP) interactions between distal PEs ( $>10 \mathrm{~kb}$ from TSS) and bivalent promoters ( $n=526$ ) were visualized as pileup plots using $\mathrm{Hi}-\mathrm{C}$ data generated in the epiblast from WT and Kmt2b/Mll2-/- E6.5 mouse embryos ${ }^{51}$. Hi-C interaction matrices were KR-balanced (balanced). f Significant (mESC H3K27me3 HiChIP data; $p<0.01$; FitHiChIP) interactions between distal PEs ( $>10$ kb from TSS) and bivalent promoters ( $n=526$ ) were visualized as pileup plots using $\mathrm{Hi}-\mathrm{C}$ data generated in CTCF-Degron mESC lines ${ }^{53}$ that were either untreated (CTCF untreated), treated with Auxin for two days (CTCF Aux (2 days)) or treated with Auxin and then washed off (CTCF Aux (washoff)). Interaction matrices were KRbalanced (balanced). In (b, d-f), HiChIP or Hi-C pairwise interactions are shown $250 \mathrm{~kb}$ up- and downstream of each PE and bivalent promoter pair. The numbers in the upper left corners correspond to "loopiness" values (center intensity normalized to the intensity in the corners).

investigated vertebrate species, PEs were located close to CGIs ${ }^{6,22}$ and linked to major developmental genes. We recently showed that orphan CGIs are an essential component of PEs that, together with TAD boundaries, enable them to precisely and specifically control the expression of developmental genes with CpGrich promoters ${ }^{9}$. We also showed that the main regulatory function of these orphan CGI is to serve as tethering elements that bring PEs and their CpG-rich target genes into physical proximity ${ }^{9}$. Furthermore, the oCGI might also contribute to the high sequence conservation of PEs by protecting them from $\mathrm{CpG}$ methylation ${ }^{9}$ and, thus, from accumulating $\mathrm{C}>\mathrm{T}$ mutations. Therefore, we propose that the association of distal enhancers with CGI might represent an ancestral regulatory mechanism in vertebrate genomes that enables the precise and specific induction 

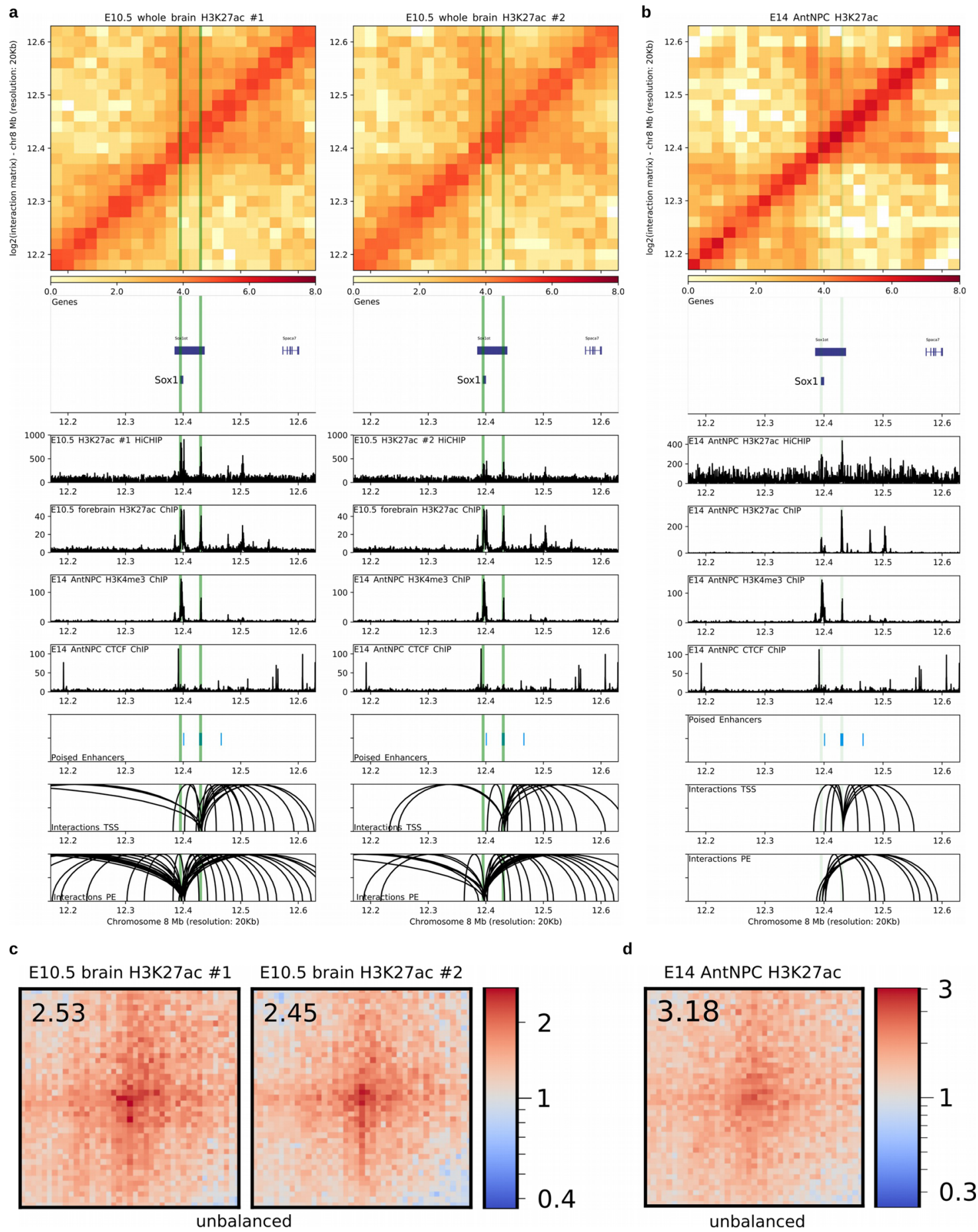

Fig. 5 Interactions between PEs and their target genes are maintained once PE becomes active in either AntNPC or the embryonic mouse brain. a, $b$ H3K27ac HiChIP and ChIP-seq profiles generated in E10.5 mouse brains (a; two biological replicates) and AntNPC (b; both replicates merged), respectively, are shown around the Sox 1 locus. Both the Lhx5 TSS and the PE Sox1 ( $+35 \mathrm{~kb}$ ) are marked with green vertical lines. Upper panel: heatmap of $\log 2$ interaction intensities based on H3K27ac HiChIP data generated in E10.5 mouse brain. Two medium panels: 1D HiChIP signals for H3K27ac in E10.5 mouse brain and H3K27ac, H3K4me3, and CTCF (for potential TAD boundaries) ChIP-seq profiles in AntNPC. Lower two panels: significant ( $p<0.01$ for E10.5 mouse brains; $p<0.05$ for AntNPC; FitHiChIP) interactions were called using the H3K27ac HiChIP PETs (paired-end tags) and loops in which one of the anchors overlaps $( \pm 10 \mathrm{~kb}$ ) either the PE Sox1 ( $+35 \mathrm{~kb}$ ) or the Lhx5 TSS are shown. c, d Significant (mESC H3K27me3 HiChIP data; $p<0.01 ;$ FitHiChIP) interactions between distal PEs ( $>10 \mathrm{~kb}$ from TSS) and ESC bivalent promoters $(n=526)$ were visualized as pileup plots using H3K27ac HiChIP data generated in H3K27ac HiChIP data generated in E10.5 mouse brain (c) or AntNPC (d). HiChIP interaction matrices were coverage-normalized (unbalanced). HiChIP pairwise interactions are shown $250 \mathrm{~kb}$ up- and downstream of each PE and bivalent promoter pair. The numbers in the upper left corners correspond to "loopiness" values (center intensity normalized to the intensity in the corners). 

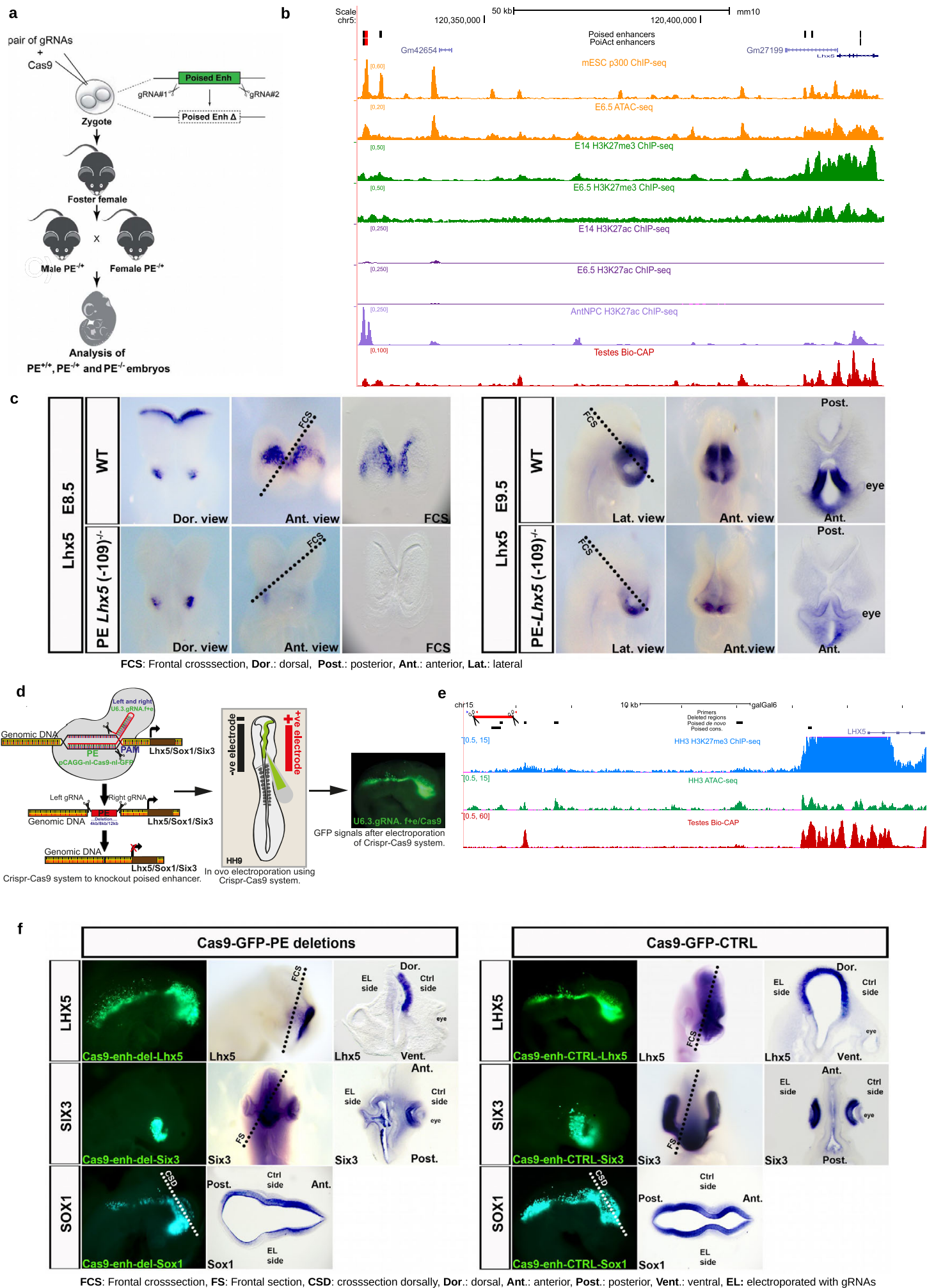

of major developmental genes within large regulatory domains ${ }^{57-59}$. Interestingly, although CGIs are considered as a vertebrate-specific genetic feature, sequences with equivalent tethering and regulatory functions might also exist in invertebrates, where they can also be important for the long-range induction of major developmental genes ${ }^{60-63}$.
As mentioned above, the orphan CGI associated with PEs act as tethering elements physically linking these distal regulatory elements with their target genes. Mechanistically, in undifferentiated ESC, this tethering function seems to be mediated by PcG complexes recruited to the CGI present both at PEs and their target gene promoters ${ }^{5,9,23,64}$. Here we show that these PE-gene 
Fig. 6 Functional relevance of PEs during in vivo brain development. a Graphical overview of the CRISPR/Cas9 experimental strategy used to generate mouse embryos with homozygous deletions of the PE Lhx5 (-109kb). b ATAC-seq and ChIP-seq (p300, H3K27me3, and H3K27ac) profiles generated in mESC and E6.5 mouse epiblast are shown around the Lhx5 locus. In addition, the Bio-CAP profiles generated in mouse testes are shown to illustrate the location of CGls in the mouse genome, as well as H3K27ac ChIP-seq profiles generated in AntNPC to illustrate the activation of PE Lhx5 ( $-109 \mathrm{~kb}$ ) in AntNPC. The genomic region deleted in mouse embryos that includes the PE $L h \times 5(-109 \mathrm{~kb})$ is highlighted in red. c RNA in situ hybridizations were performed to visualize $L h \times 5$ expression in WT and PE $L h \times 5(-109 \mathrm{~kb})^{-/-}$mouse embryos at embryonic stages E8.5 (top rows) and E9.5 (bottom rows). Five PE $L h \times 5(-109 \mathrm{~kb})^{-/-}$and four WT mouse embryos were analyzed for each developmental stage, with all PE Lhx5 (-109 kb) ${ }^{-/-}$embryos showing the reduced Lhx5 expression in the brain as compared to WT. d Graphical overview of the CRISPR/Cas9-based approach used to delete three genetically conserved PEs located within the Lhx5, Sox1, and Six3 loci (left panel). Schematic diagram of in ovo electroporation technique in chick embryo at HH9. Mosaic knockout chicken embryos were generated by unilateral co-electroporation of CAGG > nls-Cas9-nls-GFP and U6.3>PE Lhx5/Sox1/Six3gRNAf +e vectors into the brain region (medium panel). GFP expression in brain and neural tube of an embryo electroporated with the Cas9 and gRNA-GFP vectors (right panel). e ATAC-seq and H3K27me3 ChIP-seq profiles generated in HH3 chicken epiblast are shown around the Lhx5 locus. In addition, Bio-CAP profiles generated in chicken testes are also shown to illustrate the location of CGIs in the chicken genome. The genomic region deleted in chicken embryos that includes the mouse PE $L h \times 5(-109 \mathrm{~kb})$ conserved in chicken is highlighted in red. $\mathbf{f}$ RNA in situ hybridizations were performed to visualize Lhx5, Six3 or Sox1 expression in $\mathrm{HH} 14-\mathrm{HH} 16$ chicken embryos that were unilaterally electroporated with (i) Left panels: Cas9-GFP and gRNAs flanking the conserved PEs associated with Lhx5, Six3 and Sox1 (Cas9-GFP-PE deletions); (ii) Right panels: Cas9-GFP and scrambled gRNA (Cas9-GFP-CTRL). For Six3, 16 Cas9-GFP$P E$ deletion embryos were analyzed and all of them showed decreased Six 3 expression and an aberrant eye phenotype on the electroporated side. For $L$ hx5, 20 Cas9-GFP-PE deletion embryos were analyzed and 14/20 embryos showed decreased $L$ hx 5 expression on the electroporated side, while $6 / 20$ embryos showed mild or no reduction. For Sox1, 13 Cas9-GFP-PE deletion embryos were analyzed and 10/13 chicken embryos showed decreased Sox 1 expression on the electroporated side, while 3/13 embryos showed no reduction. For each of the previous three genes, we used six Cas9-GFP-CTRL chicken embryos as controls and no changes in gene expression were observed on the electroporated sides.

contacts are globally dependent on PRC1, while PRC2 seems to preferentially contribute to the interactions occurring within specific loci ${ }^{5}$. These results are in agreement with previous reports indicating that long-range interactions between PcG-loci are mediated by cPRC1 subunits ${ }^{35,45-49,65}$, with PRC2 having a lesser and indirect contribution through its capacity to recruit $\mathrm{CPRC} 1$ to its genomic targets ${ }^{66}$. CGI can serve as recruitment platforms for other proteins containing CXXC domains (e.g. TET1, CFP1, MLL2/KMT2B), which are frequently part of important chromatin regulatory complexes (e.g. Trithorax $(\operatorname{Trx} G))^{67-69}$. It was recently shown that MLL2/KMT2B, an important component of TrxG complexes, can also contribute to the $3 \mathrm{D}$ chromatin organization of bivalent genes in pluripotent cells ${ }^{51,70}$. Interestingly, here we found that MLL2/KMT2B also facilitates the interaction between PE and their bivalent target genes. Therefore, PcG and TrxG complexes might cooperate rather than antagonize each other in pluripotent cells in order to mediate homotypic chromatin interactions within PE loci that facilitate future gene induction $39,48,71,72$. In addition to PcG and TrxG complexes, 3D chromatin organization is largely dependent on the combined effects of Cohesin and CTCF, which are necessary for the formation of TADs and other large regulatory domains through a loop extrusion mechanism ${ }^{53,73-75}$. Although PEs are not directly bound by either Cohesin or CTCF in $\mathrm{ESC}^{5}$, we found that $\mathrm{PE}$-gene contacts were diminished when either of these two architectural proteins were degraded. Therefore, loop extrusion might also facilitate the physical interactions between PEs and genes located within the same TAD. This is in contrast to the role of Cohesin as a negative regulator of inter-TAD interactions between PcG-bound genes ${ }^{52}$.

Transcriptional and phenotypic robustness during development is believed to require complex regulatory landscapes whereby multiple enhancers redundantly control the expression of major cell identity genes ${ }^{76-78}$. In contrast, using ESC as an in vitro differentiation system, we previously showed that PEs can control the induction of genes involved in early brain development in a hierarchical and non-redundant manner ${ }^{5}$. Importantly, using both mouse and chicken embryos as experimental models, we have now confirmed the essential role of PEs for the proper induction of developmental genes during vertebrate embryogenesis. We propose that the privileged regulatory properties of PEs depend, at least partly, on nearby oCGI, which confer these regulatory elements with unique chromatin and topological features ${ }^{9}$. However, it is important to mention that it is still unclear, both in vitro as well as in vivo, whether the "poised" state is actually important for enhancer function. Therefore, the oCGI might confer PEs their privileged regulatory properties once they become active in differentiating cells. Lastly, it is certainly possible that not all PEs acquire their unique chromatin and topological features already in pluripotent cells and this might occur later in lineage-restricted multipotent progenitors ${ }^{79}$. Therefore, future work should elucidate the full repertoire of PEs and interrogate their function in different somatic lineages and spatiotemporal contexts.

\section{Methods}

Cell culture. $10 \mathrm{~cm}$ plates were coated with $0.1 \%$ gelatin generally overnight. Cells (E14 WT, EED-/ ${ }^{41}$ and RING1a-/-RING1b f//142 $\mathrm{mESC}$ ) were thawed and resuspended in $10 \mathrm{ml}$ of medium (serum + LIF). Standard serum + LIF medium contained $500 \mathrm{~mL}$ Knockout DMEM (Gibco 10829-018), $95 \mathrm{~mL}$ of filtered ES FBS (Gibco 16141-061), $5.9 \mathrm{~mL}$ of antibiotics (Hyclone SV30079.01), $5.9 \mathrm{~mL}$ Glutamax (Gibco 35050-038), $5.9 \mathrm{~mL}$ MEM NEAA (Gibco 11140-035), $4.7 \mathrm{~mL}$ titrated LIF (Miltenyi Biotec 130-095-777), and $1.3 \mathrm{~mL}$ Beta-mercaptoethanol $55 \mathrm{mM}$ (Gibco 21985-023). Cells were split once ( $1 / 3$ or $1 / 4)$ every two days. Ring1a-/-Ring1 $1 b^{f / / f l}$ mESC were treated with Tamoxifen (OHT; $1 \mathrm{mM}$ ) for $72 \mathrm{~h}$ right after one passage and RING1B loss was confirmed by PCR genotyping and Western Blot.

AntNPC differentiation. E14 WT ESC grown in serum + LIF were treated with 2 $\mathrm{mL}$ TrypLE Express (Life Technologies). Cells were centrifuged (160 rcf) and then resuspended in $5 \mathrm{~mL}$ of N2B27 with $0.1 \%$ of BSA (Life Technologies) to get a single cell suspension. Next, cells were counted with the BioRad TC20 cell counter and 15,000 cells $/ \mathrm{cm}^{2}$ were plated in $10 \mathrm{~mL}$ of N2B27 with $0.1 \%$ of BSA and $10 \mathrm{ng} / \mathrm{mL}$ of bFgf (PeproTech, 100-18B). Cells were differentiated for five days and media was changed every day without any PBS washings in between: Day1 $(10 \mathrm{~mL}$ of N2B27 with $0.004 \%$ of BSA and $10 \mathrm{ng} / \mathrm{mL}$ bFgf).; Day2 (10 mL of N2B27 with $0.004 \%$ of BSA, $10 \mathrm{ng} / \mathrm{mL}$ bFgf and $5 \mu \mathrm{M}$ Xav939/Wnt inhibitor (Sigma-Aldrich, x3004$5 \mathrm{mg})$ ); Day3 $(10 \mathrm{~mL}$ of N2B27 with $0.004 \%$ of BSA and $5 \mu \mathrm{M}$ Xav939/Wnt inhibitor); Day4 (10 mL of N2B27 with $0.004 \%$ of BSA and $5 \mu \mathrm{M} \mathrm{Xav939/Wnt} \mathrm{inhi-}$ bitor). On Day 5, cells were washed 1-2 with PBS, and collected for downstream analyses. Differentiation was assessed using RT-qPCR on a Light Cycler 480II comparing AntNPC d5 vs. E14 WT d0 relative gene expression levels (using the $2 \Delta \mathrm{Ct}$ method) with housekeeping gene (Eeflal), pluripotency markers

(Pou5f1/Oct4 and Nanog), mesoderm marker (T) and ectoderm markers (Six3 and $L h x 5)$. Standard deviations were calculated from technical triplicate reactions and were represented as error bars.

ATAC-seq. Embryonic tissues were extracted and resuspended into single cells ATAC-seq was essentially conducted following the protocol from Buenrostro et al. ${ }^{80}$. In short, single cells were centrifugated at $5000 \mathrm{~g}$ for $5 \mathrm{~min}$ and supernatant 
was removed. Cells were then lysed with $100 \mu \mathrm{l}$ cold lysis buffer $(10 \mathrm{mM}$ Tris- $\mathrm{HCl}$, pH 7.4, $10 \mathrm{mM} \mathrm{NaCl}, 3 \mathrm{mM} \mathrm{MgCl}_{2}, 0,1 \%$ IGEPAL CA-630) supplemented with 4 $\mu \mathrm{l}$ of protease inhibitor (1 tablet $/ 2 \mathrm{~mL}$ conc.) for at least $15 \mathrm{~min}$ on ice. Immediately after lysis, nuclei were centrifugated at $6000 \mathrm{~g}$ for $10 \mathrm{~min}$ at $4{ }^{\circ} \mathrm{C}$. The resulting pellet was resuspended in transposase reaction mix $(25 \mu \mathrm{l} 2 \mathrm{x}$ TD buffer, $10 \mu \mathrm{l}$ transposase (Illumina), $15 \mu \mathrm{l}$ nuclease-free $\mathrm{H}_{2} \mathrm{O}$ ) and incubated for $30 \mathrm{~min}$ at $37^{\circ} \mathrm{C}$. Finally, the sample was purified using Qiagen MinElute PCR purification kit according to the manufacturer's protocol

ChIP. Cells were crosslinked in $1 \%$ of formaldehyde for 10 min (rotating at RT) with subsequent quenching by glycine $(0.125 \mathrm{M}$; rotating at RT). Cells were washed twice with PBS and 1x protease inhibitor (04693159001, Roche), then flash frozen in liquid nitrogen and stored at $-80^{\circ} \mathrm{C}$. Afterwards cells were thawed for $\sim 30 \mathrm{~min}$ and lysed in $50 \mathrm{mM}$ Hepes, $140 \mathrm{mM} \mathrm{NaCl}, 1 \mathrm{mM}$ EDTA, $10 \%$ glycerol, $0.5 \% \mathrm{NP}-40$ and $0.25 \%$ TX-100 together with protease inhibitor (Lysis Buffer 1) for $10 \mathrm{~min}$ at 4 ${ }^{\circ} \mathrm{C}$ while rotating. After centrifugation $\left(5 \mathrm{~min}, 2000 \mathrm{rcf}, 4^{\circ} \mathrm{C}\right)$, the supernatant was discarded and the pellet resuspended in $10 \mathrm{mM}$ Tris, $200 \mathrm{mM} \mathrm{NaCl}, 1 \mathrm{mM}$ EDTA, $0.5 \mathrm{mM}$ EGTA, protease inhibitor (Lysis Buffer 2) and lysed for $10 \mathrm{~min}$ at $4{ }^{\circ} \mathrm{C}$ while rotating. After centrifugation $\left(5 \mathrm{~min}, 2000 \mathrm{rcf}, 4^{\circ} \mathrm{C}\right.$ ), the supernatant was discarded and the pellet was resuspended in $10 \mathrm{mM}$ Tris, $100 \mathrm{mM} \mathrm{NaCl}, 1 \mathrm{mM}$ EDTA, $0.5 \mathrm{mM}$ EGTA, $0.1 \%$ Na-Deoxycholate and $0.5 \%$ N-lauroylsarcosine with protease inhibitor (Lysis Buffer 3). Chromatin was then sonicated with ActiveMotif Sonicatior (amplitude: $25 \%$, on $=20 \mathrm{~s}$, off $=30 \mathrm{~s}, 20$ cycles). The sonicated chromatin was centrifugated for $10 \mathrm{~min}, 16,000 \mathrm{rcf}$ at $4{ }^{\circ} \mathrm{C}$. The supernatant was collected and supplemented with $10 \%$ Triton X-100. $10 \%$ of the sonicated chromatin was kept as input DNA. For each ChIP reaction, $5 \mu \mathrm{g}$ antibody were added to the remaining sonicated chromatin. ChIP samples were rotated vertically at $4{ }^{\circ} \mathrm{C}$ overnight $(12-16 \mathrm{~h})$ to bind antibody to chromatin. On the next day, $50-75 \mu \mathrm{l}$ magnetic Dynabeads (Protein G) were washed three times (3X) in $1 \mathrm{~mL}$ cold Block Solution $(0.5 \%$ BSA (w/v), 1x PBS). Antibody-bound chromatin was added to beads and inverted to mix. Then rotated vertically at $4{ }^{\circ} \mathrm{C}$ for at least $4 \mathrm{~h}$. Afterwards, bound beads were washed $5 \mathrm{X}$ in $1 \mathrm{~mL}$ cold RIPA buffer $(50 \mathrm{mM}$ Hepes, $500 \mathrm{mM} \mathrm{LiCl}, 1$ mM EDTA, $1 \%$ NP-40, 0.7\% Na-Deoxycholate). Then, samples were washed once in $1 \mathrm{~mL} \mathrm{TE}+50 \mathrm{mM} \mathrm{NaCl}$ on ice and centrifuged for $3 \mathrm{~min}$ at $1000 \mathrm{rcf}, 4^{\circ} \mathrm{C}$ to remove all remaining TE. $210 \mu$ Elution Buffer was added to the beads and DNA was eluted for $15 \mathrm{~min}$ in a thermoblock at $65^{\circ} \mathrm{C}$ with shaking (900 RPM). Samples were centrifuged ( $1 \mathrm{~min}, 16,000 \mathrm{rcf}, \mathrm{RT}$ ) and the supernatants were transferred $(\sim 200 \mu \mathrm{L})$ to fresh microfuge tubes. Both ChIP and input samples were then reverse-crosslinked and treated with RNAse and Proteinase K. Finally, DNA was extracted by phenol-chlorophorm followed by ethanol precipitation and resuspension in water. DNA content was measured using Qubit and the HS DNA Kit (Invitrogen Q32851). All antibodies used in this study have been previously reported as suitable for ChIP: H3K4me3 (39159, Active Motif), H3K27ac (39133, Active Motif), CTCF (61312, Active Motif), H3K27me3 (39155, Active Motif).

HiChIP. HiChIPs were performed as described ${ }^{33}$ with some modifications. We generally replaced the ChIP protocol with the one described above, we cut and ligated overnight (NEB T4 Ligase, \#M0202 instead of Invitrogen T4, 15224-041) and DNA was extracted with phenol-chlorophorm. For low cell numbers, we increased the centrifugation time to $30 \mathrm{~min}$ and $15 \mathrm{~min}$ after lysis, as well as $30 \mathrm{~min}$ after ligation to see a pellet more accurately. Generally, 12 cycles were used for Tn5 Nextera PCR amplification (Illumina Nextera DNA UD Indexes Kit). We aimed for $100 \mathrm{M}$ read pairs for each run on a HiSeq 2500 sequencer (Illumina), except for the AntNPC samples which were sequenced at $50 \mathrm{M}$ read depth. We used approx. $1 / 4$ of a $10 \mathrm{~cm}$ plate for each cell culture HiChIP replicate. For each murine E10.5 brain replicate, we used at least $1.5 \mathrm{M}$ cells.

\section{Generation of CRISPR/Cas9-mediated PE deletions in mice. The CRISPR/Cas9} endonuclease-mediated PE deletions were generated by the CECAD in vivo Research Facility (ivRF) by pronuclear injection of the Cas9 nuclease mRNA and protein, tracrRNA, and crRNAs into C57BL/6 zygotes ${ }^{81}$. Cas9 nuclease (Addgene \#1074181), tracrRNA (Addgene \#1072532), and custom crRNA sequences were purchased from Integrated DNA Technologies (IDT; Coralville, Iowa, USA). The animals were housed and bred under standard conditions in the CECAD ivRF under a $12 \mathrm{~h}$ light cycle, at a temperature of $22 \pm 2{ }^{\circ} \mathrm{C}, 55 \pm 5 \%$ relative humidity and with food and water ad libitum. The breedings described were approved by the Landesamt für Natur, Umwelt, und Verbraucherschutz Nordrhein-Westfalen (LANUV), Germany (animal application 84-02.04.2015.A405).

\section{Designing and molecular cloning of chicken gRNA. The template sequence} flanking the PE region for each gene of interest (Lhx5, Sox1, and Sox3) was obtained from the UCSC Genome Browser and used for gRNA design. The gRNA sequences were designed using Benchling (Supplementary Data 1). We followed the standard principles to avoid off-target effects when choosing between multiple gRNA targets for each PE and cloned into the modified U6.3>gRNA.f + e backbone (Addgene \#99139) as described in ${ }^{55}$. After cloning the gRNA into the backbone, the positive bacterial clones were identified using colony PCR with the corresponding forward and reverse gRNA oligo described in Supplementary Data 1. The resulting PCR products were analyzed by Sanger sequencing (SeqLab).
We used a control gRNA with a protospacer sequence not found in the chicken genome (GCAC-TGCTACGATCTACACC) which is already cloned into U6.3>Control.gRNA f $+\mathrm{e}$ vector, also provided by Prof. Dr. Marianne Bronner (Addgene \#99140). To validate the CRIPSR/Cas9 targeting efficiency, PCR-based genotyping was done.

Genotyping of PE deletions. Whole chicken embryos were electroporated with pCAGG $>$ nls-hCas9-nls-GFP together with either U6.3>PE $L h x 5$ gRNA f $+\mathrm{e}$, U6.3>PE_Sox1 gRNA $\mathrm{f}+\mathrm{e}$ or U6.3>PE_Six3 gRNA $\mathrm{f}+\mathrm{e}$ at stage HH9, then incubated until stage HH14-16 was reached. Live embryos were dissected in sterile PBS (1x) on ice and electroporated GFP-positive regions were isolated using surgical scissors. After isolation, neural tube and eye sections were pooled in a $1.5 \mathrm{ml}$ tube separately for each embryo and used immediately dissociated for genotyping with one volume of Lysis Buffer (LyB; $50 \mathrm{mM} \mathrm{KCL}, 10 \mathrm{mM}$ TRIS pH 8.3, $2.5 \mathrm{mM}$ $\mathrm{MgCl}_{2}, 0.45 \% \mathrm{NP} 40$ and $0.45 \%$ Tween 20$)$, containing Proteinase K (1 $\mu \mathrm{lof} 20 \mu \mathrm{g} /$ $\mu \mathrm{l}$ of Proteinase $\mathrm{K}$ for every $25 \mu \mathrm{l}$ of $\mathrm{LyB}$ ) and incubated at $55^{\circ} \mathrm{C}$ for $1 \mathrm{~h}$ with frequent shaking to mix. The lysate was then heated to $95^{\circ} \mathrm{C}$ for another $10 \mathrm{~min}$ to inactivate Proteinase K. We then tested for the presence of the PE deletions by PCR-based genotyping using the specific primers described in Supplementary Data 1

To detect the PE Lhx5 deletion in mice, genomic DNA was isolated from ear punches and analyzed by PCR using the primers shown in Supplementary Data 1 . The deletion was further confirmed by Sanger sequencing of the deletion-specific PCR products (Seqlab).

Chicken embryos. Fertilized chicken eggs (white leghorn; Gallus gallus domesticus) were obtained from a local breeder (LSL Rhein-Main) and incubated at $37^{\circ} \mathrm{C}$ and $80 \%$ humidity in a normal poultry egg incubator (Typenreihe Thermo-deLux). Following microsurgical procedures, the eggs were re-incubated until the embryos reached the desired developmental stages. The developmental progress was determined according to the staging system of $\mathrm{HH}^{82}$.

According to the relevant German legislation ("Tierschutz-Versuchsverordnung") work with non-mammalian vertebrate embryos (e.g. chicken embryos) before $2 / 3$ of their total developmental window does not require ethical approval. In all performed experiments, chicken embryos were kept no longer than stage HH14-16 (2-2.5 days), which is considerably earlier than $2 / 3$ of the chicken embryo whole developmental window (20-21 days).

In ovo electroporation. Electroporations were performed using stage HH9 chicken embryos. $3.5-4 \mathrm{~mL}$ of albumin were removed by using a medical syringe to lower the blastoderm and make the embryo accessible for manipulation. The eggs were windowed, and the extra embryonic membrane was partially removed in the region to be electroporated. For knockout experiments, $5 \mu \mathrm{g} / \mu \mathrm{l}$ pCAGG $>$ nls-hCas9-nlsGFP (Addgene \#99141) and $3 \mu \mathrm{g} / \mu \mathrm{l}$ U6.3 > Lhx5/Sox1/Six3 gRNA f + e was microinjected together with the Fast Green solution (Sigma) at a 2:1 ratio to ease the detection of the injection site of the developing neural tube and eye respectively with the help of borosilicate glass capillaries and electroporated by placing the electrodes on each side of the microinjected neural tube/eye, and five square pulses of $80 \mathrm{~V}$ within $20 \mathrm{~ms}$ width were applied to each embryo using the Intracel TSS20 OVODYNE Electroporator ${ }^{83-85}$. Control embryos were similarly electroporated with $1.5 \mu \mathrm{g} / \mu \mathrm{l}$ U6.3>Control.gRNA $\mathrm{f}+\mathrm{e}$ along with $\mathrm{pCAGG}>$ nls-hCas9-nls-GFP. Following electroporation, the eggs were sealed with medical tape and re-incubated until the desired developmental stages (HH14-16) were reached.

In situ hybridization (ISH). At the desired stages, embryos were dispatched and fixed in 4\% PFA/PBT overnight for ISH. Whole mount ISH of electroporated chicken embryos (HH14-HH16) and mutant/wt mice embryos (E8.5 and E9.5) was performed with probes against the target genes as described in ${ }^{85,86}$. For $L h x 5$, Sox 1 and Six3, T7 promoter-containing PCR products were synthesized from stage HH9-HH14 chick cDNA. The gel-purified PCR products were used as templates for synthesis of antisense RNA probes using T7 polymerase enzyme. Primers are described in Supplementary Data 1. Riboprobes were labeled with a digoxigenin RNA labeling kit (Thermofisher, AM1324). Furthermore, mutant/wt mice embryos were tested for $L h x 5$, using DIG-labeled RNA probe against mouse $L h x 5$ (986 bp) (Supplementary Data 1) by cloning the template into the $\mathrm{pCR}^{\mathrm{w} I I} \mathrm{II}$-TOPO (Thermofischer) vector, digestion with XhoI and in vitro transcription using the SP6 polymerase.

Chicken embryo sectioning and microscopy. Selected embryos were sectioned using a vibratome (Leica) at a thickness of 30-35 $\mu \mathrm{m}$. Light microscopy image were taken on a Olympus SZX16 stereomicroscope, To prepare the permanent slide, sections were embedded in Aquatex (Merck)

ChIP- \& ATAC-seq analysis. Essentially the same analysis workflows were used for ChIP-seq and ATAC-seq data. Sequencing reads were mapped with bwa-0.7.7 $\mathrm{mem}^{87}$, then converted from SAM to BAM (with samtools-1.288) and sorted (with samtools-1.2). We then removed duplicate reads (with picard-tools-2.5.0 ${ }^{89}$ ) and generated an index file (with samtools-1.2). Finally, bigWig/bedGraph files were 
generated with deepTools ${ }^{90}$ bamCoverage 2.5.7, normalized to $1 \mathrm{x}$ depth of coverage (reads per genome coverage), profiled and uploaded into the UCSC browser $^{91}$ (hub hosted on cyverse ${ }^{92}$ ). Peaks are called with macs 2.1.1.2016030993. The narrow peak mode and the corresponding default threshold $(q=0.05)$ were used to call ATAC-seq and p300 peaks. The broad peak mode and the corresponding default threshold $(q=0.1)$ were used to call H3K4me1, H3K4me3, H3K27ac, and H3K27me3 peaks. The ChIP-seq data from mouse WT in vitro pluripotent cell types (i.e. serum+LIF ESC, 2i ESC, and EpiLC) was obtained from Cruz-Molina et al. ${ }^{5}$ and Bleckwehl et al. ${ }^{94}$. Mapping statistics for our generated data are listed in Supplementary Data 1. All publically available ChIP-seq and ATAC-seq data sets used in this work, including those generated by ENCODE ${ }^{95}$, are listed in Supplementary Data 1. Multiple files of the same entity were merged using bigWigMerge.

Enhancer calling. The previous ATAC-seq and ChIP-seq peaks were used to call active, primed and poised enhancers using basic operations (INTERSECT, SUBTRACT, MERGE) available in bedtools2-2.19.0 $0^{96}$.

Mouse in vitro pluripotent cell types: Poised and active enhancers in serum + LIF ESC were previously reported in Cruz-Molina et al. ${ }^{5}$. For the previous enhancer sets, those located proximal to gene TSS $( \pm 5 \mathrm{~kb})$ were filtered out. H3K27ac, H3K4mel, and H3K27me3 broad peaks (default: $q \leq 0.1$ ) in 2i ESC and EpiLC were additionally filtered by requiring fold-enrichments over input of at least 5, 2, and 2, respectively. H3K27ac peaks were similarly identified in serum + LIF ESC. The resulting peaks were extended $\pm 1 \mathrm{~kb}$. p300 and ATAC-seq narrow peaks (default: $q \leq 0.05$ ) in $2 \mathrm{i}$ ESC and EpiLC were additionally filtered by requiring fold-enrichments over input of at least 4.

Poised enhancers-2i ESC and EpiLC ATAC-seq peaks overlapping (INTERSECT) with genomic regions enriched in both $\mathrm{H} 3 \mathrm{~K} 27 \mathrm{me} 3$ and $\mathrm{H} 3 \mathrm{~K} 4 \mathrm{mel}$ in the corresponding cell type were identified and combined with the serum+LIF PEs via UNION. Those genomic regions located proximal to gene TSS $( \pm 5 \mathrm{~kb})$ were filtered out. Then, genomic regions being enriched in $\mathrm{H} 3 \mathrm{~K} 27 \mathrm{ac}$ in any of the in vitro pluripotent cell types were SUBTRACTED (H3K27ac peaks identified in 2i, serum+LIF, and EpiLC were combined via UNION). Finally, the resulting genomic regions were MERGED to define a total of 4191 unique PEs in in vitro mouse pluripotent cells.

Active enhancers-2i ESC and EpiLC ATAC-seq peaks overlapping (INTERSECT) with genomic regions enriched in both H3K27ac and H3K4mel in the corresponding cell type were identified and combined with the serum+LIF active enhancers via UNION. Those genomic regions located proximal to gene TSS $( \pm 5 \mathrm{~kb})$ were filtered out. Then, genomic regions being enriched in $\mathrm{H} 3 \mathrm{~K} 27 \mathrm{me} 3 \mathrm{in}$ any of the in vitro pluripotent cell types were SUBTRACTED (H3K27me3 peaks identified in 2i, serum+LIF, and EpiLC were combined via UNION). Finally, the resulting genomic regions were MERGED to define a total of 14803 unique active enhancers in in vitro mouse pluripotent cells.

Primed enhancers- $\mathrm{H} 3 \mathrm{~K} 4 \mathrm{mel}$ peaks identified in 2i ESC and EpiLC were combined with the serum+LIF primed enhancers via UNION. Those genomic regions located proximal to gene TSS $( \pm 5 \mathrm{~kb})$ were filtered out. Then, genomic regions being enriched in $\mathrm{H} 3 \mathrm{~K} 27 \mathrm{me} 3$ or H3K27ac in any of the in vitro pluripotent cell types were SUBTRACTED (H3K27me3 peaks identified in 2i, serum+LIF, and EpiLC were combined via UNION; H3K27ac peaks identified in 2i, serum+LIF and EpiLC were combined via UNION). Finally, the resulting genomic regions were MERGED to define a total of 55812 primed enhancers in in vitro mouse pluripotent cells.

To avoid redundancies between the different enhancer groups, enhancers overlapping between each of the three previous categories (poised, active, and primed) were filtered out, except for those overlapping active and primed enhancers $(n=855)$, which were attributed to the active enhancer category.

PoiAct enhancers-PEs identified in the in vitro pluripotent cell types were intersected with either in vitro AntNPC H3K27ac peaks ( $\mathrm{q} \leq 0.1$; broad; foldenrichment $\geq 5$; extension $\pm 1 \mathrm{~kb}$ ) or in vivo E10.5 brain (fore-, mid- and hindbrain $)^{95} \mathrm{H} 3 \mathrm{~K} 27$ ac peaks ( $\mathrm{q} \leq 0.1$; broad; fold-enrichment $\geq 1$; in vivo). To call $\mathrm{H} 3 \mathrm{~K} 27 \mathrm{ac}$ peaks in E10.5 fore-, mid- and hindbrain, we overlapped (INTERSECT) the peaks identified in each of the two biological replicates available for each brain part (Supplementary Data 1).

Mouse E6.5 epiblast-to call in vivo mouse PEs, E6.5 epiblast ATAC-seq peaks (FC $\geq 5 ; q=0.05$; narrow peak mode) overlapping (INTERSECT) with genomic regions enriched in $\mathrm{H} 3 \mathrm{~K} 27 \mathrm{me} 3$ ( $\mathrm{FC} \geq 2$; $p \leq 0.01$; extension $\pm 1 \mathrm{~kb}$; broad peak mode) in the E6.5 epiblast were identified. Then, genomic regions enriched in $\mathrm{H} 3 \mathrm{~K} 27 \mathrm{ac}$ in either E6.5 epiblast (FC $\geq 2 ; p \leq 0.01$; broad peak mode) or in vitro pluripotent cell types (UNION of H3K27ac peaks identified in 2i ESC, serum+LIF ESC, EpiLC) were subtracted. Finally, genomic regions located proximal to gene TSS $( \pm 5 \mathrm{~kb})$ were filtered out to define a total of 3057 PEs in the mouse E6.5 epiblast.

H3K4mel was not used to define in vivo mouse PEs as ChIP-seq data for this histone mark was not available in the E6.5 epiblast. Moreover, the peak calling criteria for H3K27ac and H3K27me3 in the E6.5 epiblast were more relaxed than in the in vitro pluripotent cell types due to the overall lower quality of in vivo ChIPseq data sets. To ensure that the identified in vivo PEs are not active in pluripotent cells and due to the lower quality of the in vivo ChIP-seq data, we subtracted $\mathrm{H} 3 \mathrm{~K} 27 \mathrm{ac}$ regions identified in any of the investigated in vitro pluripotent cell types.

Human, chicken, and zebrafish-for human, chicken, and zebrafish, de novo PEs were called using similar criteria to those described for mouse cells, but we extended each H3K27me3 and H3K27ac (H3K27ac only available for human ESC) peak by $\pm 2.5 \mathrm{~kb}$ and we only considered regions located distally $(>10 \mathrm{~kb})$ from gene TSS. For calling ChIP-seq and ATAC-seq peaks we always used $\mathrm{q} \leq 0.1$. Moreover, for the p300/ATAC-seq peaks we required the following fold-enrichment over input thresholds: human $\geq 3$, chicken $\geq 3$ and zebrafish $\geq 5$. For the H3K27me3 peaks we required the following fold-enrichment over input thresholds: human $\geq 3$, chicken $\geq 1$, and zebrafish $\geq 3$. The H3K27ac peaks in human ESC were required a fold-enrichment over input threshold $\geq 3$.

All generated enhancer bed files are listed in Supplementary Data 2.

PE sets were annotated using GREAT 4.0.497 (human and mouse) and ConsensusPathDB Release 34 (15.01.2019) ${ }^{98}$ (chicken and zebrafish). The used assemblies and annotations are listed in Supplementary Data 1.

Conservation analysis. All available UCSC vertebrates were considered if they had an available liftOver chain file from mm10 (Supplementary Data 1 for all used genome builds). Specific genome build versions were used to match Ensembl TSS annotations of the species in which Bio-CAP data was available ${ }^{22}$. Identity thresholds of $50 \%$ were used.

All species depictions are royalty-free/limited licensed under the stock IDs 57341071, 1666821223, 750090523, 99158564, 182837423, 194504681 \& 1536755681 .

Non-methylated islands (NMI). NMI and Bio-CAP data were obtained from Long et al. ${ }^{22}$. Processed NMI bed files were initially used to calculate NMI to PE distances. Raw reads were downloaded from $\mathrm{GEO}^{99}$ (GSE43512), aligned using bowtie2-2.2.0 ${ }^{100}$ and profiled using deepTools bamCoverage 2.5.7. Narrow NMI peaks were called using macs 2.1.1.20160309 callpeak ( $\mathrm{q} \leq 0.01$; “-extsize 300 -mfold 1030 ").

HiChIP. Except for murine E10.5 brain samples, biological/technical replicates were merged. Reads were aligned and quality-controlled through HiC-Pro 2.10101. Initial anchor calling was conducted using macs 2.1.1.20160309 callpeak on bowtie alignments with default $\mathrm{q} \leq 0.1$ (E14 mESC H3K27me3; E10.5 brain H3K27ac) or $\mathrm{q} \leq 0.01$ (residual). The resulting peaks were passed to FitHiChIP 7.0102 (29th April 2019) for final loop calling (in L_COV mode). Basic loop quality control statistics including distance plots were conducted using diffloop $1.14 .0^{103}$. hichipper $0.7^{104}$ was used to generate bedgraph files and ChIP signal profiles. BigWig files were generated after clipping bedGraph files using bedClip. Final tracks were visualized in WashU ${ }^{105}$ or UCSC genome browsers. Loop anchors were overlapped with TSS $( \pm 7.5 \mathrm{~kb})$ and annotated using ConsensusPathDB ${ }^{98}$. Hi-C matrices were processed and normalized using cooler $0.8 .7^{106}$ ( $5 \mathrm{~kb}$ resolution), then plotted with coolpup. py 0.9.2 ${ }^{107}$ using default settings with "-padding 100". HiChIP samples were coverage-normalized (unbalanced) and Hi-C samples were KR-balanced (balanced). Loopiness values were calculated on the single center pixel normalized to corners (“-enrichment 1 -norm_corners 1 "). Single loci were visualized using HiCPlotter 0.8.1 $1^{108}$ with standard RefSeq annotations. PcG domains for looping analyses were called by overlapping significant peaks (macs2; narrow; $\mathrm{q}=0.1$ ) of $\mathrm{EED}^{109}$ and RING1b ${ }^{110}$ ChIP-seq (three replicates each against input; peaks intersected) binding sites. TAD evaluations were conducted using coordinates ${ }^{111}$ included within the HiCPlotter package lifted over from $\mathrm{mm} 9$ to $\mathrm{mm} 10$. All residual statistical analyses were conducted using custom-made scripts in $\mathrm{R}$ (3.6.0; 2019-04-26).

RNA-seq and scRNA-seq. The expression of targets genes (evaluated through sign. E14 H3K27me3 HiChIP interactions) for different poised and PoiAct enhancer sets was plotted for AntNPC and mESC RNA-seq ${ }^{5}$, as well as for blastulation, gastrulation, and neurulation samples from two scRNA-Seq data sets ${ }^{112,113}$. Expression differences between differentiation or developmental stages were evaluated using two-sided Wilcoxon tests. Processed data were obtained from the respective GEO entries. To circumvent batch effects in both plots, expression was divided by housekeeping genes (all available eukaryotic translation elongation factors and actin molecules) mean expression.

\section{Data availability}

The data that support this study are available from the corresponding authors upon reasonable request. The ChIP-seq, ATAC-seq and HiChIP data generated in this study has been deposited in the GEO repository database under accession code GSE160657. The generated enhancer sets in this study are provided in Supplementary Data 2.

All public data sets used are listed here and in Supplementary Data 1:

GSE155089, GSE65583, GSE89211, GSE41267, GSE69919, GSE41923, GSE74617, GSE73952, GSE43512, GSE124342, GSE100597, GSE98671, GSE87038, GSE38066, GSE24447, E-MTAB-7816, GSE23716, GSE66390, GSE125318, GSE76505, E-MTAB6165, GSE76687, GSE70355. The source data are provided with this paper.

\section{Code availability}

All analysis was conducted using established software wrapped in custom-written scripts. Scripts are available upon request. 
Received: 29 January 2021; Accepted: 21 June 2021;

Published online: 16 July 2021

\section{References}

1. Rada-Iglesias, A. et al. A unique chromatin signature uncovers early developmental enhancers in humans. Nature 470, 279-283 (2011).

2. Creyghton, M. P. et al. Histone H3K27ac separates active from poised enhancers and predicts developmental state. Proc. Natl Acad. Sci. USA 107, 21931-21936 (2010).

3. Entrevan, M., Schuettengruber, B. \& Cavalli, G. Regulation of genome architecture and function by polycomb proteins. Trends Cell Biol. 26, 511-525 (2016).

4. Schoenfelder, S. et al. Polycomb repressive complex PRC1 spatially constrains the mouse embryonic stem cell genome. Nat. Genet. 47, 1179-1186 (2015).

5. Cruz-Molina, S. et al. PRC2 facilitates the regulatory topology required for poised enhancer function during pluripotent stem cell differentiation. Cell. Stem Cell. 20, 689-705.e9 (2017).

6. Illingworth, R. S. et al. Orphan CpG islands identify numerous conserved promoters in the mammalian genome. PLoS Genet. 6, e1001134 (2010).

7. van Heeringen, S. J. et al. Principles of nucleation of H3K27 methylation during embryonic development. Genome Res. 24, 401-410 (2014).

8. Li, Y. et al. Genome-wide analyses reveal a role of Polycomb in promoting hypomethylation of DNA methylation valleys. Genome Biol. 19, 18 (2018).

9. Pachano, T. et al. Orphan $\mathrm{CpG}$ islands amplify the regulatory activity of poised enhancers and dictate the responsiveness of their target genes. Nat. Genet. Jun 28, https://doi.org/10.1038/s41588-021-00888-x (2021)

10. Hackett, J. A. \& Surani, M. A. Regulatory principles of pluripotency: from the ground state up. Cell Stem Cell 15, 416-430 (2014).

11. Morgani, S., Nichols, J. \& Hadjantonakis, A. The many faces of pluripotency: in vitro adaptations of a continuum of in vivo states. BMC Dev. Biol. 17, 7 (2017).

12. $\mathrm{Wu}, \mathrm{J}$. et al. The landscape of accessible chromatin in mammalian preimplantation embryos. Nature 534, 652-657 (2016).

13. Liu, X. et al. Distinct features of H3K4me3 and H3K27me3 chromatin domains in pre-implantation embryos. Nature 537, 558-562 (2016).

14. Zheng, $\mathrm{H}$. et al. Resetting epigenetic memory by reprogramming of histone modifications in mammals. Mol. Cell 63, 1066-1079 (2016).

15. Maezawa, S. et al. Polycomb protein SCML2 facilitates H3K27me3 to establish bivalent domains in the male germline. Proc. Natl Acad. Sci. USA 115, 4957-4962 (2018).

16. Hiller, M. et al. Computational methods to detect conserved non-genic elements in phylogenetically isolated genomes: application to zebrafish. Nucleic Acids Res. 41, e151 (2013).

17. Bejerano, G. et al. Ultraconserved elements in the human genome. Science 304, 1321-1325 (2004)

18. Dickel, D. E. et al. Ultraconserved enhancers are required for normal development. Cell 172, 491-499.e15 (2018)

19. Elnitski, L. \& Ovcharenko, I. The hypothesis of ultraconserved enhancer dispensability overturned. Genome Biol. 19, 57 (2018).

20. Romero, I. G. et al. A panel of induced pluripotent stem cells from chimpanzees: a resource for comparative functional genomics. Elife 4, e07103 (2015).

21. Kaaij, L. J. T. et al. Enhancers reside in a unique epigenetic environment during early zebrafish development. Genome Biol. 17, 146 (2016).

22. Long, H. K. et al. Epigenetic conservation at gene regulatory elements revealed by non-methylated DNA profiling in seven vertebrates. Elife 2, e00348 (2013).

23. $\mathrm{He}$, J. et al. Kdm2b maintains murine embryonic stem cell status by recruiting PRC1 complex to CpG islands of developmental genes. Nat. Cell Biol. 15, 373-384 (2013).

24. Blackledge, N. P. et al. Variant PRC1 complex-dependent H2A ubiquitylation drives PRC2 recruitment and polycomb domain formation. Cell 157, $1445-1459$ (2014).

25. Chen, S., Jiao, L., Liu, X., Yang, X. \& Liu, X. A Dimeric Structural Scaffold for PRC2-PCL Targeting to CpG Island Chromatin. Mol. Cell. 77, 1265-1278.e7 (2020).

26. Habibi, E. et al. Whole-genome bisulfite sequencing of two distinct interconvertible DNA methylomes of mouse embryonic stem cells. Cell Stem Cell 13, 360-369 (2013).

27. Zylicz, J. J. et al. Chromatin dynamics and the role of G9a in gene regulation and enhancer silencing during early mouse development. Elife 4, e09571 (2015).

28. Farcas, A. M. et al. KDM2B links the Polycomb Repressive Complex 1 (PRC1) to recognition of $\mathrm{CpG}$ islands. Elife 1, e00205 (2012).

29. Boulard, M., Edwards, J. R. \& Bestor, T. H. FBXL10 protects polycomb-bound genes from hypermethylation. Nat. Genet 47, 479-485 (2015).
30. Rada-Iglesias, A. et al. Epigenomic annotation of enhancers predicts transcriptional regulators of human neural crest. Cell Stem Cell 11, 633-648 (2012).

31. Belaghzal, H., Dekker, J. \& Gibcus, J. H. Hi-C. 2.0: An optimized Hi-C procedure for high-resolution genome-wide mapping of chromosome conformation. Methods 123, 56-65 (2017).

32. Bonev, B. et al. Multiscale 3D genome rewiring during mouse neural development. Cell 171, 557-572.e24 (2017).

33. Mumbach, M. R. et al. HiChIP: efficient and sensitive analysis of proteindirected genome architecture. Nat. Methods 13, 919-922 (2016).

34. Mikkelsen, T. S. et al. Genome-wide maps of chromatin state in pluripotent and lineage-committed cells. Nature 448, 553-560 (2007).

35. Kundu, S. et al. Polycomb repressive complex 1 generates discrete compacted domains that change during differentiation. Mol. Cell 65, 432-446.e5 (2017).

36. McLaughlin, K. et al. DNA methylation directs polycomb-dependent 3D genome re-organization in naive pluripotency. Cell Rep. 29, 1974-1985.e6 (2019).

37. Zhang, Y. et al. Dynamic epigenomic landscapes during early lineage specification in mouse embryos. Nat. Genet. 50, 96-105 (2018).

38. Joshi, O. et al. Dynamic reorganization of extremely long-range promoterpromoter interactions between two states of pluripotency. Cell Stem Cell 17, 748-757 (2015).

39. Gentile, C. et al. PRC2-associated chromatin contacts in the developing limb reveal a possible mechanism for the atypical role of PRC2 in HoxA gene expression. Dev. Cell. 50, 184-196.e4 (2019).

40. Loubiere, V. et al. Widespread activation of developmental gene expression characterized by PRC1-dependent chromatin looping. Sci. Adv. 6, eaax4001 (2020).

41. Schoeftner, S. et al. Recruitment of PRC1 function at the initiation of X inactivation independent of PRC2 and silencing. EMBO J. 25, 3110-3122 (2006).

42. Endoh, M. et al. Polycomb group proteins Ring1 A/B are functionally linked to the core transcriptional regulatory circuitry to maintain ES cell identity. Development 135, 1513-1524 (2008).

43. Leeb, M. et al. Polycomb complexes act redundantly to repress genomic repeats and genes. Genes Dev. 24, 265-276 (2010).

44. Ferrari, K. J. et al. Polycomb-dependent H3K27me1 and H3K27me2 regulate active transcription and enhancer fidelity. Mol. Cell. 53, 49-62 (2014).

45. Isono, K. et al. SAM domain polymerization links subnuclear clustering of PRC1 to gene silencing. Dev. Cell 26, 565-577 (2013).

46. Plys, A. J. et al. Phase separation of polycomb-repressive complex 1 is governed by a charged disordered region of CBX2. Genes Dev. 33, 799-813 (2019).

47. Tatavosian, R. et al. Nuclear condensates of the polycomb protein chromobox 2 (CBX2) assemble through phase separation. J. Biol. Chem. 294, 1451-1463 (2019).

48. Pachano, T., Crispatzu, G. \& Rada-Iglesias, A. Polycomb proteins as organizers of 3D genome architecture in embryonic stem cells. Brief. Funct. Genomics 18, 358-366 (2019).

49. Kent, S. et al. Phase-separated transcriptional condensates accelerate targetsearch process revealed by live-cell single-molecule imaging. Cell Rep. 33, 108248 (2020).

50. Schuettengruber, B., Bourbon, H., Di Croce, L. \& Cavalli, G. Genome regulation by polycomb and trithorax: 70 years and counting. Cell 171, 34-57 (2017).

51. Xiang, Y. et al. Epigenomic analysis of gastrulation identifies a unique chromatin state for primed pluripotency. Nat. Genet. 52, 95-105 (2020).

52. Rhodes, J. D. P. et al. Cohesin disrupts polycomb-dependent chromosome interactions in embryonic stem cells. Cell Rep. 30, 820-835.e10 (2020).

53. Nora, E. P. et al. Targeted degradation of CTCF decouples local insulation of chromosome domains from genomic compartmentalization. Cell 169, 930-944.e22 (2017).

54. Kishi, Y. \& Gotoh, Y. Regulation of chromatin structure during neural development. Front Neurosci. 12, 874 (2018).

55. Gandhi, S., Piacentino, M. L., Vieceli, F. M. \& Bronner, M. E. Optimization of CRISPR/Cas9 genome editing for loss-of-function in the early chick embryo. Dev. Biol. 432, 86-97 (2017).

56. Carl, M., Loosli, F. \& Wittbrodt, F. Six3 inactivation reveals its essential role for the formation and patterning of the vertebrate eye. Development 129, 4057-4063 (2002)

57. Kikuta, H. et al. Genomic regulatory blocks encompass multiple neighboring genes and maintain conserved synteny in vertebrates. Genome Res. 17, 545-555 (2007).

58. Akalin, A. et al. Transcriptional features of genomic regulatory blocks. Genome Biol. 10, R38 (2009).

59. Harmston, N. et al. Topologically associating domains are ancient features that coincide with Metazoan clusters of extreme noncoding conservation. Nat. Commun. 8, 441 (2017) 
60. Mahmoudi, T., Katsani, K. R. \& Verrijzer, C. P. GAGA can mediate enhancer function in trans by linking two separate DNA molecules. EMBO J. 21, 1775-1781 (2002).

61. Calhoun, V. C., Stathopoulos, A. \& Levine, M. Promoter-proximal tethering elements regulate enhancer-promoter specificity in the Drosophila Antennapedia complex. Proc. Natl Acad. Sci. USA 99, 9243-9247 (2002).

62. Calhoun, V. C. \& Levine, M. Long-range enhancer-promoter interactions in the Scr-Antp interval of the Drosophila Antennapedia complex. Proc. Natl Acad. Sci. USA 100, 9878-9883 (2003).

63. Engström, P. G., Ho Sui, S. J., Drivenes, O., Becker, T. S. \& Lenhard, B. Genomic regulatory blocks underlie extensive microsynteny conservation in insects. Genome Res. 17, 1898-1908 (2007).

64. Perino, M. et al. MTF2 recruits Polycomb Repressive Complex 2 by helicalshape-selective DNA binding. Nat. Genet 50, 1002-1010 (2018).

65. Boyle, S. et al. A central role for canonical PRC1 in shaping the $3 \mathrm{D}$ nuclear landscape. Genes Dev. 34, 931-949 (2020).

66. Cao, R. et al. Role of histone $\mathrm{H} 3$ lysine 27 methylation in polycomb-group silencing. Science 298, 1039-1043 (2002).

67. Blackledge, N. P. \& Klose, R. CpG island chromatin: a platform for gene regulation. Epigenetics 6, 147-152 (2011)

68. Deaton, A. M. \& Bird, A. CpG islands and the regulation of transcription. Genes Dev. 25, 1010-1022 (2011).

69. Wachter, E. et al. Synthetic CpG islands reveal DNA sequence determinants of chromatin structure. Elife 3, e03397 (2014).

70. Mas, G. et al. Promoter bivalency favors an open chromatin architecture in embryonic stem cells. Nat. Genet. 50, 1452-1462 (2018).

71. Kondo, T. et al. Polycomb potentiates meis 2 activation in midbrain by mediating interaction of the promoter with a tissue-specific enhancer. Dev. Cell 28, 94-101 (2014).

72. Pu, L. \& Sung, Z. R. PcG and trxG in plants-friends or foes. Trends Genet. 31, 252-262 (2015).

73. Fudenberg, G. et al. Formation of chromosomal domains by loop extrusion. Cell Rep. 15, 2038-2049 (2016).

74. Schwarzer, W. et al. Two independent modes of chromatin organization revealed by cohesin removal. Nature 551, 51-56 (2017).

75. Rao, S. S. P. et al. Cohesin loss eliminates all loop domains. Cell 171, 305-320. e24 (2017).

76. Perry, M. W., Boettiger, A. N., Bothma, J. P. \& Levine, M. Shadow enhancers foster robustness of Drosophila gastrulation. Curr. Biol. 20, 1562-1567 (2010).

77. Cannavò, E. et al. Shadow enhancers are pervasive features of developmental regulatory networks. Curr. Biol. 26, 38-51 (2016).

78. Osterwalder, M. et al. Enhancer redundancy provides phenotypic robustness in mammalian development. Nature 554, 239-243 (2018).

79. Minoux, M. et al. Gene bivalency at Polycomb domains regulates cranial neural crest positional identity. Science 355, eaal2913 (2017).

80. Buenrostro, J. D., Giresi, P. G., Zaba, L. C., Chang, H. Y. \& Greenleaf, W. J. Transposition of native chromatin for fast and sensitive epigenomic profiling of open chromatin, DNA-binding proteins and nucleosome position. Nat Methods 10, 1213-1218 (2013).

81. Chu, V. T. et al. Efficient CRISPR-mediated mutagenesis in primary immune cells using CrispRGold and a C57BL/6 Cas9 transgenic mouse line. Proc. Natl Acad. Sci. USA 113, 12514-12519 (2016).

82. Hamburger, V. \& Hamilton, H. L. A series of normal stages in the development of the chick embryo. 1951. Dev. Dyn. 195, 231-272 (1992).

83. Dai, F., Yusuf, F., Farjah, G. H. \& Brand-Saberi, B. RNAi-induced targeted silencing of developmental control genes during chicken embryogenesis. Dev. Biol. 285, 80-90 (2005).

84. Scaal, M., Gros, J., Lesbros, C. \& Marcelle, C. In ovo electroporation of avian somites. Dev. Dyn. 229, 643-650 (2004).

85. Rehimi, R. et al. Epigenomics-based identification of major cell identity regulators within heterogeneous cell populations. Cell Rep. 17, 3062-3076 (2016).

86. Nieto, H., Patel, D. G. \& Wilkinson, M. A. In situ hybridization analysis of chick embryos in whole mount and tissue sections. Methods Cell Biol. 51, 219-235 (1996).

87. Li, H. \& Durbin, R. Fast and accurate short read alignment with BurrowsWheeler transform. Bioinformatics 25, 1754-1760 (2009).

88. Li, H. et al. The sequence alignment/map format and SAMtools. Bioinformatics 25, 2078-2079 (2009).

89. Broad Institute. "Picard Tools." Broad Institute, GitHub repository. http:// broadinstitute.github.io/picard/ (Accessed: 23 Jun 2016).

90. Ramírez, F., Dündar, F., Diehl, S., Grüning, B. A. \& Manke, T. deepTools: a flexible platform for exploring deep-sequencing data. Nucleic Acids Res. 42, W187-W191 (2014). (Web Server issue).

91. Haeussler, M. et al. The UCSC Genome Browser database: 2019 update. Nucleic Acids Res. 47, D853-D858 (2019).

92. Merchant, N. et al. The iPlant collaborative: cyberinfrastructure for enabling data to discovery for the life sciences. PLoS Biol. 14, e1002342 (2016).
93. Zhang, Y. et al. Model-based analysis of ChIP-Seq (MACS). Genome Biol. 9, R137 (2008)

94. Bleckwehl, T. et al. Enhancer priming by H3K4 methylation safeguards germline competence. bioRxiv https://doi.org/10.1101/2020.07.07.192427 (2020).

95. ENCODE Project Consortium. An integrated encyclopedia of DNA elements in the human genome. Nature 489, 57-74 (2012).

96. Quinlan, A. R. BEDTools: the swiss-army tool for genome feature analysis. Curr. Protoc. Bioinform. 47, 11.12.1-34 (2014).

97. McLean, C. Y. et al. GREAT improves functional interpretation of cisregulatory regions. Nat. Biotechnol. 28, 495-501 (2010).

98. Herwig, R., Hardt, C., Lienhard, M. \& Kamburov, A. Analyzing and interpreting genome data at the network level with ConsensusPathDB. Nat. Protoc. 11, 1889-1907 (2016)

99. Barrett, T. et al. NCBI GEO: archive for functional genomics data sets-update Nucleic Acids Res. 41, D991-D995 (2013). (Database issue).

100. Langmead, B. \& Salzberg, S. L. Fast gapped-read alignment with Bowtie 2. Nat Methods 9, 357-359 (2012).

101. Servant, N. et al. HiC-Pro: an optimized and flexible pipeline for $\mathrm{Hi}-\mathrm{C}$ data processing. Genome Biol. 16, 259 (2015).

102. Bhattacharyya, S., Chandra, V., Vijayanand, P. \& Ay, F. Identification of significant chromatin contacts from HiChIP data by FitHiChIP. Nat. Commun. 10, 4221 (2019)

103. Lareau, C. A. \& Aryee, M. J. diffloop: a computational framework for identifying and analyzing differential DNA loops from sequencing data. Bioinformatics 34, 672-674 (2018).

104. Lareau, C. A. \& Aryee, M. J. hichipper: a preprocessing pipeline for calling DNA loops from HiChIP data. Nat. Methods 15, 155-156 (2018).

105. Li, D., Hsu, S., Purushotham, D., Sears, R. L. \& Wang, T. WashU Epigenome Browser update 2019. Nucleic Acids Res. 47, W158-W165 (2019).

106. Abdennur, N. \& Mirny, L. A. Cooler: scalable storage for Hi-C data and other genomically labeled arrays. Bioinformatics 36, 311-316 (2020).

107. Flyamer, I. M., Illingworth, R. S. \& Bickmore, W. A. Coolpup.py: versatile pileup analysis of Hi-C data. Bioinformatics 36, 2980-2985 (2020).

108. Akdemir, K. C. \& Chin, L. HiCPlotter integrates genomic data with interaction matrices. Genome Biol. 16, 198 (2015)

109. Zhang, W. et al. The BAF and PRC2 complex subunits Dpf2 and Eed antagonistically converge on Tbx3 to control ESC differentiation. Cell. Stem Cell. 24, 138-152.e8 (2019).

110. Tavares, L. et al. RYBP-PRC1 complexes mediate H2A ubiquitylation at polycomb target sites independently of PRC2 and H3K27me3. Cell 148, 664-678 (2012).

111. Dixon, J. R. et al. Topological domains in mammalian genomes identified by analysis of chromatin interactions. Nature 485, 376-380 (2012).

112. Mohammed, H. et al. Single-cell landscape of transcriptional heterogeneity and cell fate decisions during mouse early gastrulation. Cell Rep. 20, 1215-1228 (2017).

113. Pijuan-Sala, B. et al. A single-cell molecular map of mouse gastrulation and early organogenesis. Nature 566, 490-495 (2019).

\section{Acknowledgements}

The authors thank the Rada-Iglesias lab members for insightful comments and critical reading of the manuscript, Elisabeth Kirst and Janine Altmüller (Cologne Center for Genomics; University of Cologne (UoC)) for technical assistance with next-generation sequencing, and the Regional Computing Center of the UoC (RRZK) for providing computing time on the DFG-funded High-Performance Computing (HPC) system CHEOPS, as well as support. We acknowledge and appreciate the CECAD in vivo Research Facility (Branko Zevnik) for the generation and maintenance of the deleted PE Lhx5 mouse line. We also thank Anton Wutz and Miguel Vidal for generously providing us with the EED-/- and RING1a-/-RING1 $b^{f / / \mathrm{fl}} \mathrm{mESC}$ lines. Work in the Rada-Iglesias laboratory was supported by CMMC intramural funding (Germany), the German Research Foundation (DFG) (Research Grant RA 2547/1-3), "Programa STAR-Santander Universidades, Campus Cantabria Internacional de la convocatoria CEI 2015 de Campus de Excelencia Internacional" (Spain), the Spanish Ministry of Science, Innovation and Universities (Research Grant PGC2018-095301-B-I00) and the European Research Council (ERC CoG"PoisedLogic“; 862022). Giuliano Crispatzu is supported by funding within the CRU329 (DFG 386793560). Rizwan Rehimi is supported by funding within the SFB829 (DFG 73111208).

\section{Author contributions}

G.C., R.R.R., A.R.I. conducted most research and prepared the manuscript. G.C. conducted bioinformatical analysis and HiChIPs. R.R.R. deleted PEs in HH9 chicken, did in situs and HH3 chicken ChIP-/ATAC-seqs. C.X. and H.B. deleted PEs in E8.5 and E9.5 mice. HB and RRR did in situ in mice deletions. G.C. and TP did PcG-null ChIPs and molecular evaluations. T.B. cultured 2i cells and differentiated EpiLC with 
subsequent ChIP-seqs. S.C.M. technically assisted with initial HiChIP experiments. E.M. provided support with in vivo mouse experiments.

\section{Funding}

Open Access funding enabled and organized by Projekt DEAL.

\section{Competing interests}

The authors declare no competing interests.

\section{Additional information}

Supplementary information The online version contains supplementary material available at https://doi.org/10.1038/s41467-021-24641-4.

Correspondence and requests for materials should be addressed to G.C. or A.R-I.

Peer review information Nature Communications thanks Darío Lupiáñez and the anonymous reviewers for their contribution to the peer review of this work. Peer reviewer reports are available.

Reprints and permission information is available at http://www.nature.com/reprints

Publisher's note Springer Nature remains neutral with regard to jurisdictional claims in published maps and institutional affiliations.

(c) (i) Attribution 4.0 International License, which permits use, sharing, adaptation, distribution and reproduction in any medium or format, as long as you give appropriate credit to the original author(s) and the source, provide a link to the Creative Commons license, and indicate if changes were made. The images or other third party material in this article are included in the article's Creative Commons license, unless indicated otherwise in a credit line to the material. If material is not included in the article's Creative Commons license and your intended use is not permitted by statutory regulation or exceeds the permitted use, you will need to obtain permission directly from the copyright holder. To view a copy of this license, visit http://creativecommons.org/ licenses/by/4.0/.

(C) The Author(s) 2021 